\title{
Coupling of fast and Alfvén waves in a straight bounded magnetic field with density stratification
}

\author{
I. Arregui, R. Oliver, and J. L. Ballester
}

\begin{abstract}
Departament de Física, Universitat de les Illes Balears, 07122 Palma de Mallorca, Spain e-mail: arregi@hubble.uib.es; ramon.oliver@uib.es;dfsjlb@@uib.es
\end{abstract}

Received 8 October 2002 / Accepted 14 February 2003

\begin{abstract}
The theoretical understanding of the linear standing or propagating magnetohydrodynamic waves in a variety of solar coronal structures is far from complete since analytical solutions to the linearised MHD equations can only be found for very simple magnetic configurations. In this paper, we use a numerical code to solve the linear fast and Alfvén wave equations in a very simple, bounded magnetic configuration that incorporates two features that are not usually considered in similar works, namely the longitudinal magnetic field component and wave propagation in the longitudinal direction $\left(k_{y} \neq 0\right)$. We use a numerical code (Arregui et al. 2001) that has been modified by including a staggered mesh that allows us to properly capture the spatial behaviour of solutions to the wave equations. Coupling between fast and Alfvén modes has been studied in detail and it has been found that it does not take place when the longitudinal field component is zero and the frequency of the fast mode is outside the Alfvén continuum with the same spatial structure along field lines. Under these circumstances, fast modes retain their global spatial behaviour and are also characterised by $\omega^{2}$ varying linearly with $k_{y}^{2}$, such as in a uniform medium (although here the Alfvén speed changes exponentially in the direction normal to field lines). Regarding mode coupling, its main feature is the blend of fast and Alfvén solutions with close frequencies in some modes with a mixture of their properties, namely discontinuities or jumps around certain magnetic surfaces (such as in pure Alfvén waves), global spatial distribution of the normal velocity component and non-zero density perturbations (such as in fast waves).
\end{abstract}

Key words. magnetohydrodynamics (MHD) - methods: numerical - Sun: oscillations - Sun: magnetic fields

\section{Introduction}

The theoretical study of the magnetohydrodynamic (MHD) modes of oscillation of coronal magnetic structures has been the subject of vigorous efforts in the last years. Recent high resolution observations obtained by both ground-based instruments and those onboard space satellites have provided new evidence about the presence of oscillations in different coronal magnetic structures and have therefore increased the need for improvements in the theoretical modeling of wave phenomena in these structures. Existing theoretical studies on standing and propagating MHD waves in coronal magnetic configurations show that analytical solutions to the linearised wave equations can only be obtained for very simple magnetic structures. In the case of the theoretical MHD modeling of linear waves in a homogeneous plasma, the eigenmode problem can be reduced to a purely algebraic form that allow one to obtain simple solutions to the wave equations. However, in most cases the complexity of the considered equilibrium leads to a set of complicated partial differential equations that in general do not allow for exact analytical solutions but require a numerical treatment. The situation is even more complicated when the partial differential equations are coupled.

Send offprint requests to: J. L. Ballester, e-mail: dfsjlb@@uib.es
In the context of MHD waves in coronal arcades, Oliver et al. (1993) studied analytically the modes of oscillation of a potential arcade for different density profiles and boundary conditions. This work was later extended by considering the MHD modes of oscillation of non-potential arcades with no gravity (Oliver et al. 1996). Terradas et al. (1999) obtained numerical solutions for a fully magnetostatic arcade with gravity and concluded that a necessary, although not sufficient, requirement for fast mode oscillations to be confined in the corona is that the Alfvén speed must increase with height. Ruderman et al. (1997) studied the resonant absorption of MHD waves in a potential arcade by considering two-dimensional magnetic plasma configurations with a purely poloidal magnetic field. In all these works it was assumed that the equilibrium configuration is invariant with respect to the axis of the arcade, that the equilibrium magnetic field is purely poloidal, i.e. with no magnetic field component in the longitudinal direction, and that there is no wave propagation along the arcade axis. There is one exception to this assertion: Tirry \& Poedts (1998) considered the solutions to the coupled fast and Alfvén modes in a potential arcade without a longitudinal magnetic field component although with wave propagation in the longitudinal direction. They solved the wave equations in flux coordinates by means of a series expansion that allowed them to conclude that under 
these conditions, fast and Alfvén continuum modes do not exist independently. However, these authors did not consider the spatial structure of the eigenfunctions, nor the case in which the longitudinal magnetic field component is different from zero.

More recently, Arregui et al. (2001) derived the linear MHD wave equations for a force-free, sheared magnetic field configuration and have developed a numerical code to solve the resulting system of coupled partial differential equations. The accuracy of the numerical code was checked by numerically solving two cases for which analytical or simple numerical solutions exist (namely a straight magnetic field and a potential arcade, both with no longitudinal magnetic field component and no propagation in the longitudinal direction). Our purpose in this paper is to use that numerical code to study the properties of linear MHD waves in more complicated magnetic configurations by including a longitudinal magnetic field component and also by considering wave propagation in the longitudinal direction. Here, a straight, bounded magnetic field is taken in what must be a first step towards the investigation of the MHD modes of coronal arcades and loops.

The interaction of fast and Alfvén modes in a straight and purely poloidal magnetic field have been investigated by means of time-dependent simulations by a number of authors. For example, Wright \& Rickard (1995) derived two criteria for the efficient excitation of an Alfvén resonance in a cavity driven by a broadband spectrum. Mann et al. (1995) considered the excitation of Alfvén waves by fast modes in the magnetospheric cavity and observed that fine scales develop for long times because of phase mixing. On the other hand, fast and Alfvén mode coupling has also been investigated in the context of MHD wave excitation by footpoint motions (Berghmans \& Tirry 1997; Tirry \& Berghmans 1997; De Groof et al. 2002; De Groof \& Goossens 2002) and the importance of such mode coupling for the dissipation of energy has been stressed. In contrast, in this work we do not consider the time evolution of fast and Alfvén perturbations and the transfer of energy between these waves but investigate the interaction between the fast and Alfvén normal modes of oscillation, that in turn provides us with a physical basis for the understanding of the dynamics of the system.

The work presented here bears some similarities to Halberstadt \& Goedbloed (1993) and Goedbloed \& Halberstadt (1994) who modeled a coronal loop by means of a rectangular box. However, these authors did not consider the fast mode and only obtained approximate solutions for the Alfvén mode with the help of asymptotic expansions. On the other hand, our approach consists of solving the full MHD wave equations in a cold plasma, which allows us to obtain the full spatial structure of the fast and Alfvén modes as well as to describe their coupling.

The layout of the paper is as follows. In Sect. 2 the equilibrium configuration is described and the linear MHD wave equations are derived. These equations, in the form of two coupled second order differential equations, govern Alfvén continuum modes and fast global modes. The numerical method used in solving the wave equations is described in Sect. 3. It turns out that our original numerical code requires some modifications to properly compute the spatial structure of solutions.

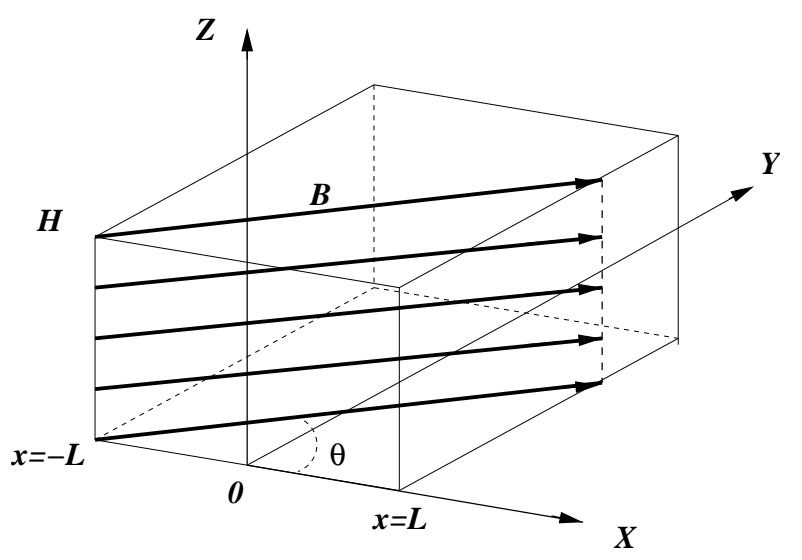

Fig. 1. Schematic view of the equilibrium magnetic field. The plasma is confined in an infinitely long box with sides at $x= \pm L, z=0$ and $z=H$.

The results of these modifications are also shown. The effect of the longitudinal magnetic field component and of the longitudinal wavenumber on the oscillatory properties of fast and Alfvén modes are presented in Sect. 4. Finally, in Sect. 5 conclusions are drawn.

\section{Basic equations}

\subsection{Equilibrium configuration}

We consider a cold plasma in a horizontal, straight and uniform magnetic field. The magnetic field is contained in the $x y$-plane of a Cartesian system of coordinates $(x, y, z)$ and is inclined with respect to the $x$-direction (see Fig. 1),

$\boldsymbol{B}=\left(B_{x}, B_{y}, 0\right)$,

where $B_{x}$ and $B_{y}$ are the constant components of the magnetic field and can be written as

$B_{x}=B \cos \theta$,

$B_{y}=B \sin \theta$,

with $B=\left(B_{x}^{2}+B_{y}^{2}\right)^{1 / 2}$ the magnitude of the magnetic field and $\theta$ the angle of inclination with respect to the $x$-direction, $\theta=\arctan \left(B_{x} / B_{y}\right)$.

The equilibrium is taken to be inhomogeneous in the vertical, $z$-direction by means of an equilibrium density which is a function of the vertical coordinate, $\rho=\rho(z)$, so that the Alfvén speed is $v_{\mathrm{A}}^{2}(z)=B^{2} / \mu \rho$. The freedom in selecting the vertical density profile allows us to consider an Alfvén speed changing with height as

$v_{\mathrm{A}}^{2}(z)=v_{\mathrm{A} 0}^{2} \exp \left[-(2-\delta) \frac{z}{L}\right]$,

$\delta$ being a parameter that determines the rate of change of the Alfvén speed with height from the value $v_{\mathrm{A} 0}$ at $z=0$.

Also, the plasma is assumed to be contained in a box limited by rigid plates at $x= \pm L$ and $z=0, H$ (Fig. 1).

The equilibrium magnetic field given by Eq. (1) is susceptible to be expressed in terms of a vector potential $\boldsymbol{A}=A(x, z) \hat{\boldsymbol{e}}_{y}$,

$\boldsymbol{B}=\nabla A \times \hat{\boldsymbol{e}}_{y}+B_{y} \hat{\boldsymbol{e}}_{y}$, 
where $\hat{\boldsymbol{e}}_{y}$ is the unit vector in the $y$-direction and the flux function $A(x, z)$ is given by

$A(x, z)=-B_{x} z+A_{0}$.

In this expression, $A_{0}$ is the value of $A$ at $z=0$. Equation (6) indicates that the magnetic surfaces (i.e. the surfaces of constant flux function) are planes of constant $z$.

\subsection{Magnetohydrodynamic linear and adiabatic wave equations}

Linear, adiabatic MHD perturbations in a cold plasma are governed by the equation (Oliver et al. 1993)

$-\rho \omega^{2} \boldsymbol{v}=\frac{1}{\mu}\{\nabla \times[\nabla \times(\boldsymbol{v} \times \boldsymbol{B})]\} \times \boldsymbol{B}$,

where $\boldsymbol{v}$ is the plasma velocity and $\boldsymbol{B}$ is the unperturbed magnetic field. For a proper description of the modes of oscillation it is convenient to project the different vectors appearing in Eq. (7) and also the components of this equation onto an adequate basis of vectors. The physically significant expressions are obtained by projecting all vectors in the directions defined at each point by the unit vectors

$\hat{\boldsymbol{e}}_{\mathrm{n}}=\frac{\nabla A}{B_{x}}=-\hat{\boldsymbol{e}}_{z}$,

normal to magnetic surfaces,

$\hat{\boldsymbol{e}}_{\|}=\frac{\boldsymbol{B}}{B}=\left(\frac{B_{x}}{B}, \frac{B_{y}}{B}, 0\right)$,

parallel to magnetic field lines, and

$\hat{\boldsymbol{e}}_{\perp}=\hat{\boldsymbol{e}}_{\|} \times \hat{\boldsymbol{e}}_{\mathrm{n}}=\left(-\frac{B_{y}}{B}, \frac{B_{x}}{B}, 0\right)$,

tangent to magnetic surfaces and perpendicular to field lines.

In the cold plasma $(\beta=0)$ approximation the perturbed velocity along the equilibrium magnetic field, $\boldsymbol{B}$, vanishes and the slow mode is absent. For this reason, the third component of Eq. (7) yields $v_{\|}=0$.

On the other hand, since the equilibrium quantities do not depend on the $y$-coordinate, we can Fourier analyse the perturbed velocity with respect to this coordinate and make it proportional to $\exp \left(i k_{y} y\right)$. This results in the following pair of second order partial differential equations for the remaining components of the velocity,

$$
\begin{aligned}
-\frac{\omega^{2}}{v_{\mathrm{A}}^{2}} v_{\mathrm{n}} & =\frac{B_{x}^{2}}{B^{2}} \frac{\partial^{2} v_{\mathrm{n}}}{\partial x^{2}}+2 \frac{B_{x} B_{y}}{B^{2}} i k_{y} \frac{\partial v_{\mathrm{n}}}{\partial x}-\frac{B_{y}^{2}}{B^{2}} k_{y}^{2} v_{\mathrm{n}}+\frac{\partial^{2} v_{\mathrm{n}}}{\partial z^{2}} \\
& +\frac{B_{y}}{B} \frac{\partial^{2} v_{\perp}}{\partial x \partial z}-\frac{B_{x}}{B} i k_{y} \frac{\partial v_{\perp}}{\partial z} \\
-\frac{\omega^{2}}{v_{\mathrm{A}}^{2}} v_{\perp} & =\frac{\partial^{2} v_{\perp}}{\partial x^{2}}-k_{y}^{2} v_{\perp}+\frac{B_{y}}{B} \frac{\partial^{2} v_{\mathrm{n}}}{\partial x \partial z}-\frac{B_{x}}{B} i k_{y} \frac{\partial v_{\mathrm{n}}}{\partial z} .
\end{aligned}
$$

Equations (11) and (12) constitute a set of two linear coupled partial differential equations for the components of the velocity in the directions normal $\left(v_{\mathrm{n}}\right)$ and perpendicular $\left(v_{\perp}\right)$ to the equilibrium magnetic field and govern the oscillatory properties of fast and Alfvén modes in our configuration.

For $k_{y}=0$ and $B_{y}=0$ the two components of the wave equations are decoupled. Then, Eq. (11) describes fast modes, whose velocity is in the normal direction $\left(v_{\mathrm{n}} \neq 0, v_{\perp}=0\right)$, whereas Eq. (12) describes Alfvén waves, whose perturbed velocity lies in the perpendicular direction $\left(v_{\mathrm{n}}=0, v_{\perp} \neq 0\right)$. The basic properties of these solutions in the present equilibrium are described in Arregui et al. (2001). Regarding other, nonzero values of the longitudinal wavenumber and magnetic field component, the above partial differential equations indicate that both $v_{\mathrm{n}}$ and $v_{\perp}$ are real for $k_{y}=0, B_{y} \neq 0$. For $k_{y} \neq 0, B_{y}=0$ one velocity component $\left(v_{\mathrm{n}}\right.$, say) is real and the other one $\left(v_{\perp}\right)$ is purely imaginary. And, finally, for $k_{y} \neq 0, B_{y} \neq 0$ both velocity components are complex and so can be expressed as

$v_{\mathrm{n}}(x, z)=\left|v_{\mathrm{n}}(x, z)\right| \mathrm{e}^{i \phi_{\mathrm{n}}(x, z)}$

and

$v_{\perp}(x, z)=\left|v_{\perp}(x, z)\right| \mathrm{e}^{i \phi_{\perp}(x, z)}$,

with $\left|v_{\mathrm{n}}(x, z)\right|$ and $\phi_{\mathrm{n}}(x, z)$ the modulus and phase of $v_{\mathrm{n}}$ (and similarly for $\left.v_{\perp}\right)$. These two expressions have a simple physical interpretation and tell us that the full spatio-temporal dependence of the velocity components is of the form $\left|v_{\mathrm{n}}(x, z)\right| \cos \left(\phi_{\mathrm{n}}(x, z)+\right.$ $\left.\omega t+k_{y} y\right)$.

To solve the coupled system of Eqs. (11) and (12) some boundary conditions have to be imposed. It can be easily shown that Eqs. (11) and (12) are fourth order in $x$, but only second order in $z$. Therefore, the proper solutions will be obtained by considering four boundary conditions in the $x$-direction and two boundary conditions in the $z$-direction. For the sake of simplicity, we impose

$v_{\mathrm{n}}=0, v_{\perp}=0 \quad$ at $\quad x= \pm L$

and

$v_{\mathrm{n}}=0 \quad$ at $\quad z=0, H / L$.

Concerning $v_{\perp}$ at $z=0, H / L$, no boundary conditions have to be considered there, but in order to obtain solutions to Eqs. (11) and (12) using finite differences some restriction must be placed on this velocity component. The vanishing of the derivative in the direction normal to the boundary is chosen by imposing

$\frac{\partial v_{\perp}}{\partial z}=0 \quad$ at $\quad z=0, H / L$

\section{Numerical method}

The numerical code we use was developed by Oliver et al. (1996) to solve the fast and slow mode equations in a twodimensional equilibrium with no gravity. Later, Arregui et al. (2001) derived the linear cold MHD wave equations for a general two-dimensional force-free magnetic configuration with a longitudinal magnetic field component and including longitudinal wave propagation, although in that work they only checked 

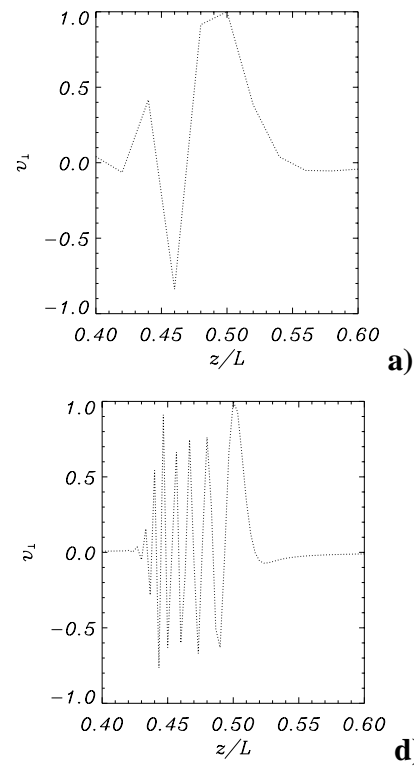

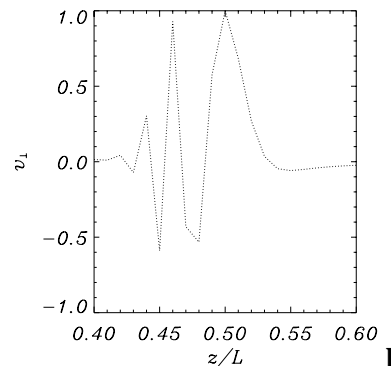

b)

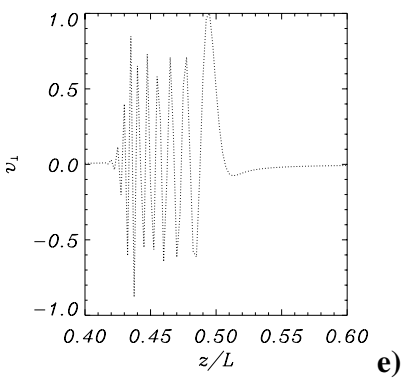

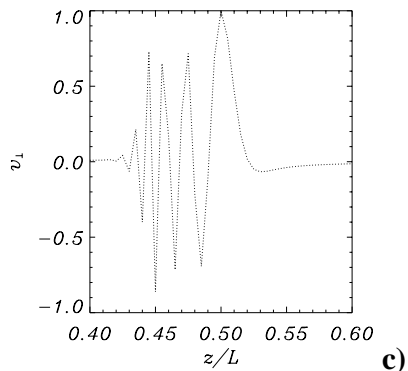

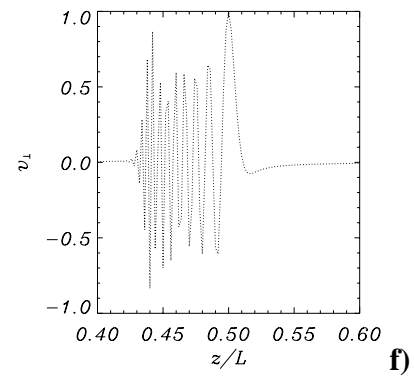

Fig. 2. Spatial structure of an Alfvén continuum mode with a singularity at $z / L=0.5$ in a straight magnetic field configuration with inclination angle $\theta=30^{\circ}, H / L=1$ and $\delta=6$ (this parameter gives the rate of change of $v_{\mathrm{A}}$ with height, see Eq. (4)). In all figures the longitudinal wavenumber is $k_{y}=0$. The numerical code described in Arregui et al. (2001) has been used with a grid of $N_{x}=51$ points and a) $N_{z}=51$, b) $N_{z}=101$, c) $N_{z}=201$, d) $N_{z}=301$, e) $N_{z}=401$ and f) $N_{z}=501$ points. The oscillations in these plots are numerical and come from the inability of the code for adequately recovering the spatial structure of Alfvén modes around the singular layer.

the goodness of the code by solving two simple problems with $B_{y}=0$ and $k_{y}=0$. This code was designed to study linear waves in more complex force-free fields and, therefore, the logical next step is to consider the case in which $B_{y} \neq 0$ and/or $k_{y} \neq 0$. We start by writing the two partial differential equations subject to be discretised in the code. These equations, written in terms of derivatives with respect to two generalised $\psi$ - and $\chi$-coordinates (which map the region $-L \leq x \leq L$, $0 \leq z \leq H$ in which the numerical solution is computed), are

$a_{11} \frac{\partial^{2} v_{\mathrm{n}}}{\partial \psi^{2}}+b_{11} \frac{\partial^{2} v_{\mathrm{n}}}{\partial \psi \partial \chi}+c_{11} \frac{\partial^{2} v_{\mathrm{n}}}{\partial \chi^{2}}+d_{11} \frac{\partial v_{\mathrm{n}}}{\partial \psi}+e_{11} \frac{\partial v_{\mathrm{n}}}{\partial \chi}$

$+f_{11} v_{\mathrm{n}}+a_{12} \frac{\partial^{2} v_{\perp}}{\partial \psi^{2}}+b_{12} \frac{\partial^{2} v_{\perp}}{\partial \psi \partial \chi}+c_{12} \frac{\partial^{2} v_{\perp}}{\partial \chi^{2}}+d_{12} \frac{\partial v_{\perp}}{\partial \psi}$

$+e_{12} \frac{\partial v_{\perp}}{\partial \chi}+f_{12} v_{\perp}=\omega^{2} v_{\mathrm{n}}$

and

$a_{21} \frac{\partial^{2} v_{\mathrm{n}}}{\partial \psi^{2}}+b_{21} \frac{\partial^{2} v_{\mathrm{n}}}{\partial \psi \partial \chi}+c_{21} \frac{\partial^{2} v_{\mathrm{n}}}{\partial \chi^{2}}+d_{21} \frac{\partial v_{\mathrm{n}}}{\partial \psi}+e_{21} \frac{\partial v_{\mathrm{n}}}{\partial \chi}$

$+f_{21} v_{\mathrm{n}}+a_{22} \frac{\partial^{2} v_{\perp}}{\partial \psi^{2}}+b_{22} \frac{\partial^{2} v_{\perp}}{\partial \psi \partial \chi}+c_{22} \frac{\partial^{2} v_{\perp}}{\partial \chi^{2}}+d_{22} \frac{\partial v_{\perp}}{\partial \psi}$

$+e_{22} \frac{\partial v_{\perp}}{\partial \chi}+f_{22} v_{\perp}=\omega^{2} v_{\perp}$.

The expressions for the complex coefficients of these two partial differential equations can be found in Arregui et al. (2001). For the numerical solution of Eqs. (18) and (19) the derivatives are substituted by appropriate finite difference expressions. The region under consideration is a rectangle with sides parallel to the $\psi$ - and $\chi$-axes, covered by a mesh of $N_{\psi} \times N_{\chi}$ points in which approximations to the functions $v_{\mathrm{n}}$ and $v_{\perp}$ are computed.
At the point $\left(\psi_{i}, \chi_{j}\right)$ the first and second derivatives of these functions are approximated in the usual manner (Mitchell \& Griffiths 1980; Ames 1992). For the purpose of obtaining numerical solutions to Eqs. (11) and (12) in the equilibrium given by Eqs. (1)-(4), Cartesian coordinates have been chosen by setting $\psi$ and $\chi$ as follows

$$
\begin{array}{ll}
\psi=z / L, & 0 \leq \psi \leq H / L, \\
\chi=x / L, & -1 \leq \chi \leq 1 .
\end{array}
$$

The discretisation process leaves us with a complex eigenvalue problem, which is solved to obtain the frequency and twodimensional velocity distribution using complex arithmetics (Arregui et al. 2001).

Nevertheless, it turns out that our numerical code requires some modifications before it can be applied to this kind of problem since it does not properly capture the spatial structure of continuum Alfvén modes. This is illustrated in Fig. 2, which shows the perpendicular velocity component of an Alfvén mode in a configuration with $\theta \neq 0^{\circ}$ and with no longitudinal wavenumber. The various solutions in this figure have been obtained with an increasing number of points in the $z$-direction $\left(N_{z}\right)$, which should allow us to better reproduce the singular character of the mode about the resonant magnetic surface. However, instead of getting a finer resolution of the singularity as $N_{z}$ is increased, a large number of numerical oscillations about the resonant surface are obtained.

In order to avoid this problem a staggered grid is used for the elimination of spurious numerical oscillations in Alfvén continuum modes. The staggered mesh method is a widely used technique in Computational Fluid Dynamics for the solution of problems involving pressure-velocity couplings that lead in 


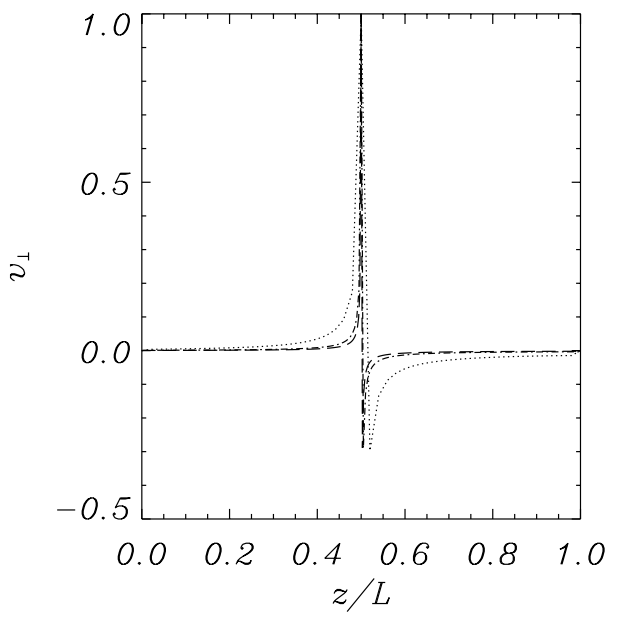

Fig. 3. Spatial profile of $v_{\perp}$ for the Alfvén continuum mode with a singular surface at $z_{\mathrm{A}}=0.5 \mathrm{~L}$. The parameters of the equilibrium configuration are $\delta=6, \theta=30^{\circ}$ and $H / L=1$. These solutions have been computed using $k_{y}=0, N_{x}=51$ (number of points in the $x$-direction) and $N_{z}=51$ (dotted line), $N_{z}=201$ (dash-dotted line) and $N_{z}=351$ (dashed line).

some cases to one or more spurious oscillatory solutions superimposed on the physical solution (see e.g. Fletcher 1988; Hoffmann \& Chiang 1989). This method implies that each dependent variable is evaluated at its own grid. The variables in the system are staggered in such a way that the locations of values and their derivatives are interlaced. In our case, $v_{\mathrm{n}}$ and $v_{\perp}$ must be located on interlaced space intervals (e.g. $v_{\mathrm{n}}$ exists on integer grid levels, while $v_{\perp}$ exists on half-integer grid levels). Further details on the implementation of this technique can be obtained from the authors.

Next, we show some solutions computed with the numerical code modified with the implementation of the staggered mesh. For the computation of the numerical solutions, a configuration with $\delta=6, H / L=1$ and an angle of inclination $\theta=30^{\circ}$ has been chosen. Figure 3 shows the vertical dependence of $v_{\perp}$ for three solutions that correspond to the same Alfvén continuum mode computed with a different number of mesh points. It can be clearly seen that an increase in the number of points in the vertical direction produces a narrower shape of the eigenfunction around the position of the ideal singular layer, as expected. When comparing these solutions to those shown in Fig. 2, the improvement with respect to the solutions obtained without the staggered mesh arrangement becomes evident. It can also be appreciated that when $B_{y} \neq 0$ the shape of the eigenfunction around the singular layer differs form the Dirac- $\delta$ function that appears when $B_{y}=0$ (Arregui et al. 2001). In addition, it can be concluded that for the computation of Alfvén continuum modes a large number of points in the singular direction is required, as expected.

\section{Numerical results}

In this section, numerical results obtained with the modified code, i.e. using a staggered mesh, are presented. As has already been stated, Eqs. (11) and (12) form a set of two coupled partial differential equations describing fast global and
Alfvén continuum modes. It is important to point out here that first of all we are interested in studying the oscillatory properties of these modes under the influence of a longitudinal magnetic field component $\left(B_{y} \neq 0\right)$ and longitudinal propagation $\left(k_{y} \neq 0\right)$. The features of the two oscillatory modes are governed both by their mutual coupling, which is associated to the interchange of properties between the modes, and by the values of $B_{y}$ and $k_{y}$. Therefore, our plan is to start from the case $B_{y}=0, k_{y}=0$ (in which fast and Alfvén modes are decoupled) and then to study the modification of the two modes when one of these parameters, or both, is different from zero. One must have in mind that for $B_{y} \neq 0$, or $k_{y} \neq 0$ or both simultaneously, fast and Alfvén modes are now coupled and so there are no longer pure fast and pure Alfvén solutions. If the frequencies of fast modes do not lie in the Alfvén continuum, the modes have mixed properties but in most cases they are predominantly fast or predominantly Alfvénic. In this case ordinary coupling occurs (Sects. 4.1 and 4.2). When the frequencies of fast modes lie inside the Alfvén continuum, resonant coupling takes place; this is studied in Sect. 4.3.

\subsection{Alfvén continuum modes}

For $B_{y}=0$ and $k_{y}=0$, wave Eqs. (11) and (12) reduce to the simple form

$$
\begin{aligned}
& -\frac{\omega^{2}}{v_{\mathrm{A}}^{2}} v_{\mathrm{n}}=\frac{\partial^{2} v_{\mathrm{n}}}{\partial x^{2}}+\frac{\partial^{2} v_{\mathrm{n}}}{\partial z^{2}} \\
& -\frac{\omega^{2}}{v_{\mathrm{A}}^{2}} v_{\perp}=\frac{\partial^{2} v_{\perp}}{\partial x^{2}}
\end{aligned}
$$

Equation (23) describes Alfvén continuum modes, decoupled from fast modes. Distribution theory (Tataronis et al. 1998) yields a solution in terms of the delta function $\delta\left(z-z_{\mathrm{A}}\right)$, where $z=z_{\mathrm{A}}$ is the singular surface, multiplied by a function of $x$. After imposing the boundary condition for $v_{\perp}$, Eq. (15), we obtain

$v_{\perp}=C \sin \left(k_{x} x\right) \delta\left(z-z_{\mathrm{A}}\right)$

with $C$ and arbitrary constant, $k_{x}=n_{x} \pi / 2 L$ and $n_{x}$ an integer that gives the number of extrema of $v_{\perp}$ in the $x$-direction. The frequency is given by

$\omega=k_{x} v_{\mathrm{A}}\left(z_{\mathrm{A}}\right)=\frac{n_{x} \pi}{2 L} v_{\mathrm{A}}\left(z_{\mathrm{A}}\right)$

\subsubsection{Frequency}

In this section the dependence of the Alfvén continuum mode frequency with the longitudinal component of the equilibrium magnetic field and with the longitudinal wavenumber is shown. From the full Eqs. (11) and (12) it is clear that the expression for the Alfvén continuum mode frequency given by Eq. (25) is not valid when $B_{y} \neq 0$ and/or $k_{y} \neq 0$. Despite the absence of pure Alfvén modes when coupling appears, in the following we keep using this term because of the dominant Alfvénic character of the solutions considered in this section. The same applies to fast modes in Sect. 4.2. 
Halberstadt \& Goedbloed (1993) obtained a similar set of equations in their study of the modes of oscillation in an inclined, straight magnetic field. Their procedure for deriving the Alfvén continuum mode frequency consists of assuming that Alfvén continuum modes are characterised by infinitely rapid variations in the direction perpendicular to magnetic surfaces, the $z$-direction in our equilibrium configuration. Hence, continuum modes must obey (Halberstadt \& Goedbloed 1993) the so-called Alfvén ordering

$\partial_{z} v_{\mathrm{n}} \gg v_{\mathrm{n}}$.

By applying this ordering to Eq. (11) we get

$\frac{\partial v_{\mathrm{n}}}{\partial z} \simeq-\frac{B_{y}}{B} \frac{\partial v_{\perp}}{\partial x}+\frac{B_{x}}{B} i k_{y} v_{\perp}$.

Now, by inserting this expression into Eq. (12) leads to the following equation

$-\frac{\omega^{2}}{v_{\mathrm{A}}^{2}} v_{\perp}=\frac{B_{x}^{2}}{B^{2}} \frac{\partial^{2} v_{\perp}}{\partial x^{2}}+2 \frac{B_{x} B_{y}}{B^{2}} i k_{y} \frac{\partial v_{\perp}}{\partial x}-\frac{B_{y}^{2}}{B^{2}} k_{y}^{2} v_{\perp}$,

which is the equation for Alfvén continuum modes. Equation (28) gives the behavior of the regular part of the solution in the singular magnetic surface and has to be solved under the boundary conditions $v_{\perp}(x=-L)=v_{\perp}(x=L)=0$.

Now different cases can be considered. In the case $B_{y} \neq 0$, $k_{y}=0$, Eq. (28) is transformed into

$-\frac{\omega^{2}}{v_{\mathrm{A}}^{2}} v_{\perp}=\frac{B_{x}^{2}}{B^{2}} \frac{\partial^{2} v_{\perp}}{\partial x^{2}}$

which after applying the boundary conditions leads to a sinusoidal variation of the regular part of $v_{\perp}$ (identical to that in Eq. (24)) and to

$\omega=k_{x} \frac{B_{x}}{B} v_{\mathrm{A}}\left(z_{\mathrm{A}}\right)=\frac{n_{x} \pi}{2 L} \cos \theta v_{\mathrm{A}}\left(z_{\mathrm{A}}\right)$

for the frequencies of Alfvén continuum modes in an inclined field with $k_{y}=0$.

By comparing Eqs. (25) and (30) it can be seen that the effect of a longitudinal magnetic field component is to decrease the frequency of Alfvén continuum modes by a factor $B_{x} / B=$ $\cos \theta$. This does not come as a surprise since the term $k_{x} B_{x} / B$ in Eq. (30) is simply $k_{\|}$.

Equation (30) is an analytical approximation and our aim here is to obtain numerical results by solving the full set of coupled Eqs. (11) and (12). Figure 4a shows the evolution of the frequency with the inclination angle for modes with $n_{x}=$ $1,2,3$, where $n_{x}$ gives the number of extrema in the $x$-direction, as in the case $B_{y}=0$. The agreement between the numerical solution and the approximate formula (30) is perfect.

Next, we consider the influence of the longitudinal wavenumber on the Alfvén continuum mode frequencies. For $B_{y}=0, k_{y} \neq 0$, Eq. (28) reduces to

$-\frac{\omega^{2}}{v_{\mathrm{A}}^{2}} v_{\perp}=\frac{\partial^{2} v_{\perp}}{\partial x^{2}}$

Equation (31) is the same as Eq. (23) (for the case $B_{y}=0$, $k_{y}=0$ ), which tells us that the longitudinal wavenumber does
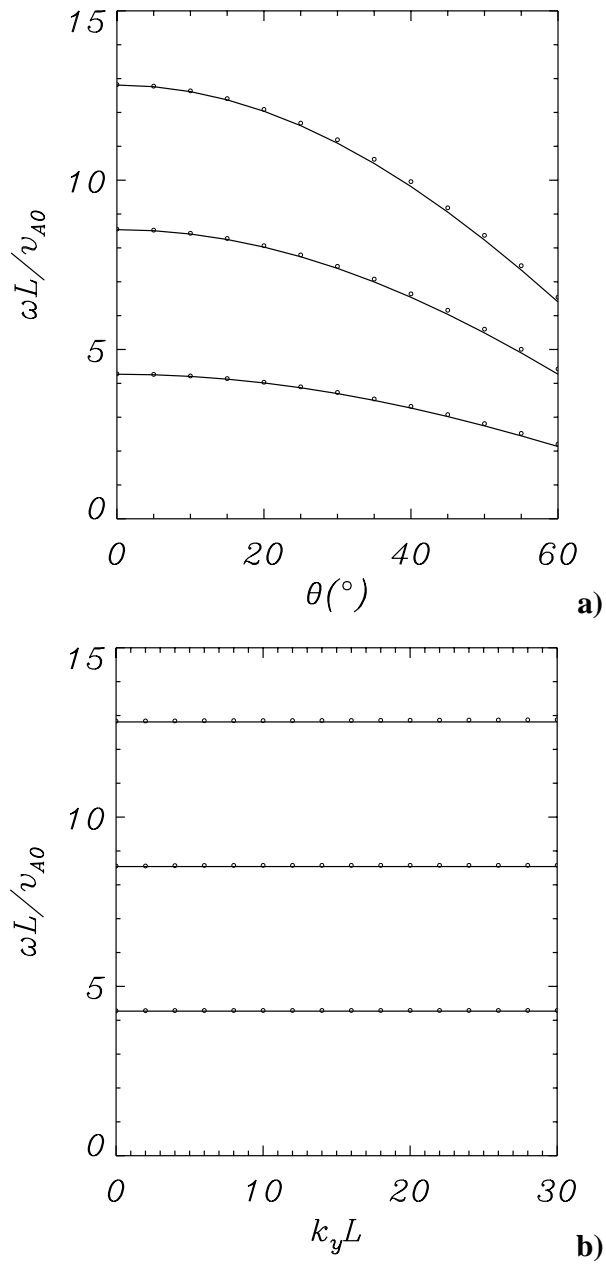

Fig. 4. Plot of the Alfvén continuum frequency as a function of a) the inclination angle in degrees for $k_{y}=0$ and $\mathbf{b}$ ) the longitudinal wavenumber with $B_{y}=0$. Solid lines represent the analytical predictions given by a) Eq. (30) and b) Eq. (25). These solutions correspond to Alfvén continuum modes with $n_{x}=1,2,3$ and a singular surface at $z_{\mathrm{A}}=0.5 L$ in a configuration with $\delta=6$ and $H / L=1$. The computational mesh is made of $N_{x}=51$ and $N_{z}=401$ points.

not affect the frequency of Alfvén continuum modes in a purely transverse magnetic field, so the frequency of Alfvén continuum modes with $B_{y}=0, k_{y} \neq 0$ is given by Eq. (25). In addition, Eq. (31) also shows that the regular part of $v_{\perp}$ is sinusoidal in $x$.

This analytical prediction is next checked by plotting the frequency of the first three Alfvén continuum modes $\left(n_{x}=1\right.$, 2,3 ) against $k_{y}$ (Fig. 4b). The numerical solutions show that the frequency of these modes is almost independent of the longitudinal wavenumber, in good agreement with the previous discussion leading to Eq. (31).

We finally study the case $B_{y} \neq 0, k_{y} \neq 0$. The solution to the differential equation Eq. (28) can be easily obtained after imposing the boundary condition given by Eq. (15) and has the following form,

$v_{\perp}\left(x, z=z_{\mathrm{A}}\right)=C \mathrm{e}^{-i \beta x} \sin \frac{n_{x} \pi}{2 L} x$ 

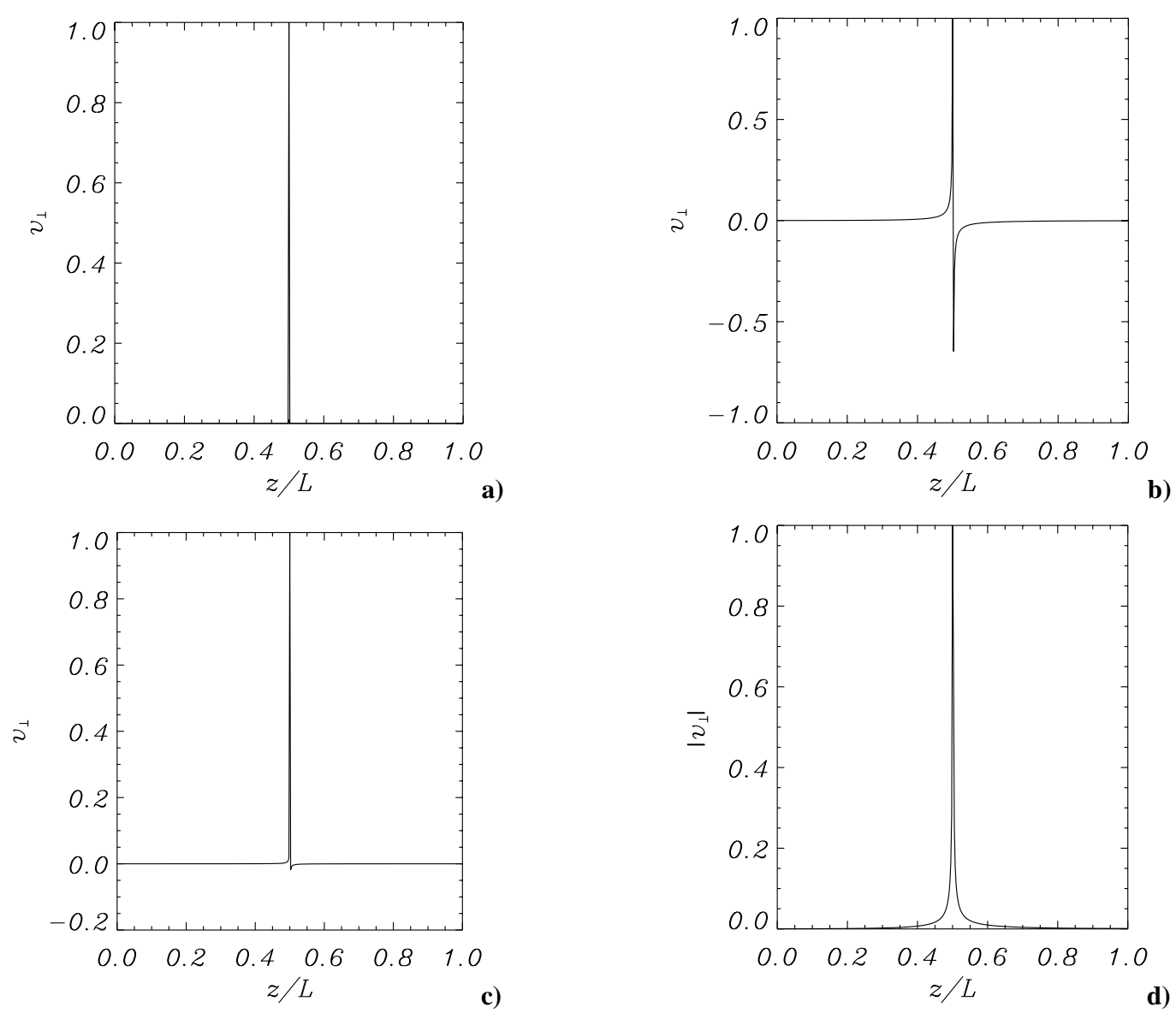

Fig. 5. Spatial profile of $v_{\perp}$ at $x / L=0$ for the Alfvén continuum mode with $z_{\mathrm{A}}=0.5 L$ for different values of $B_{y}$ and $k_{y} L$, showing the change in the singular part of the eigenfunction. a) $\theta=0^{\circ}, k_{y} L=0$, b) $\theta=0^{\circ}, k_{y} L=5$, c) $\theta=10^{\circ}, k_{y} L=0$ and d) $\theta=10^{\circ}, k_{y} L=5$. These solutions have been computed using a mesh of $N_{x}=51$ and $N_{z}=401$ points in the $x$ - and $z$-directions in a straight field configuration with $\delta=6$ and $H / L=1$.

with $C$ an arbitrary constant and $\beta$ the so-called ballooning factor, which produces a modulation of the eigenfunction in the magnetic surfaces when both $B_{y}$ and $k_{y}$ are different form zero,

$\beta=\frac{B_{y} k_{y}}{B_{x}}=\tan \theta k_{y}$.

Equation (32) is similar to Eq. (41) in Halberstadt \& Goedbloed (1993). In addition, the frequency is given by Eq. (30) and so the longitudinal wavenumber has no influence on $\omega$ independently of the angle $\theta$. This fact has been checked numerically to a high precision.

\subsubsection{Spatial structure}

The presence of a longitudinal magnetic field component and/or a longitudinal wavenumber not only affects the frequency of Alfvén continuum modes but also their spatial structure.

For $B_{y}=0$ and $k_{y}=0$, Alfvén continuum modes computed numerically with our finite difference code are characterised by $v_{\mathrm{n}} \simeq 0$ in the whole computational domain and $v_{\perp} \simeq 0$ everywhere except on the singular surface (see Arregui et al. 2001). This is a consequence of the two-dimensional spatial structure of Alfvén solutions, for which the perpendicular velocity component is given by a regular part in the $x$-direction and a singular part in the direction of the inhomogeneity in the equilibrium, i.e. the $z$-direction. Here, we exclusively concentrate on the structure of $v_{\perp}$ since our calculations always show that $v_{\mathrm{n}}$ is negligible in comparison with the perpendicular velocity component.

We first consider the singular part of Alfvén continuum modes and keep $k_{y}=0$, while gradually increasing the angle $\theta$ (Figs. 5c and 3). We see that the perpendicular velocity component loses its $\delta$-function shape and shows the expected $1 / s$ profile with a delta contribution, in agreement with Goedbloed (1983), Goossens \& Poedts (1992). The previous is true for any value of the magnetic field inclination angle, $\theta$, although this effect becomes more remarkable for larger values of this parameter. On the other hand, a similar behaviour of $v_{\perp}$ is found when the equilibrium configuration is poloidal $(\theta=0)$ and $k_{y}$ is increased (see Fig. 5b). In addition, the $1 / s$ contribution in the perpendicular velocity component is more noticeable now. We finally turn our attention to the case of a configuration with inclined magnetic field and with longitudinal wave propagation (Fig. 5d). A comparison of the shape of $v_{\perp}$ in this case with the shape in previous cases is now difficult because the perturbed velocity is a complex variable and so its modulus has been plotted. This means that the $1 / s$ profile, which results in positive and negative values of $v_{\perp}$ on both sides of the singularity, is not so easy to appreciate, although the spatial variation 


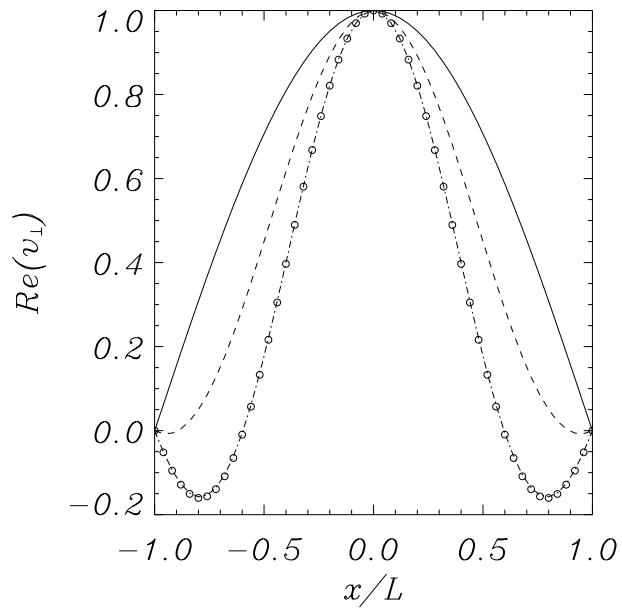

Fig. 6. Non-singular spatial profile of $v_{\perp}$ for some Alfvén solutions with a singular surface at $z_{\mathrm{A}}=0.5 \mathrm{~L}$. Continuous line: $\theta=0^{\circ}$, $k_{y} L=0$; dashed line: $\theta=10^{\circ}, k_{y} L=5$; dash-dotted line: $\theta=10^{\circ}$, $k_{y} L=15$. Circles represent the approximate analytical solution given by Eqs. (32) and (33).

of the perpendicular velocity component in Fig. $5 \mathrm{~d}$ can be well fitted by a term proportional to $|1 / s|$.

Next, the regular part of the perpendicular velocity component is considered. Our computations show that, to a very high precision, this part of the solution is sinusoidal independently of the value of $k_{y}$ and $B_{y}$; this result is in good agreement with the predictions obtained from the Alfvén ordering in Sect. 4.1.1. Nevertheless, when both these parameters are non-zero, the real and imaginary parts of $v_{\perp}$ display a shorter-scale modulation along the resonant magnetic surface (see Fig. 6 for $\operatorname{Re}\left(v_{\perp}\right)$ ). This behaviour is more evident for greater values of the product of $B_{y}$ and $k_{y}$ and is also in agreement with Eqs. (32) and (33), derived with the help of the Alfvén ordering. For a more in-depth description of this issue see Halberstadt \& Goedbloed (1993) (and also Goedbloed \& Halberstadt 1994), who showed that the spatial structure of Alfvén modes is affected by the so-called ballooning contribution, which is a modulation of Alfvén modes on different magnetic surfaces.

We finish our study of Alfvén modes by studying the velocity structure of one of these modes in an equilibrium with longitudinal magnetic field and longitudinal propagation. The moduli of the two velocity components (not shown) present the above described regular part with a sinusoidal shape together with a singular part with a $1 / s$ contribution. Note that, according to Eq. (32), the modulus of $v_{\perp}$ on the singular surface is simply $C \sin n_{x} \pi x / 2 L$ and thus is free from the ballooning contribution that gives rise to the modulations visible in Fig. 6. The spatial distribution of the phases looks rather awkward, but it must be stressed that these quantities are of interest only near the resonant surface, where the oscillatory velocity is different from zero. Cuts of $\phi_{\perp}$ and $\phi_{\mathrm{n}}$ along a magnetic surface near the singular layer show that both phases vary almost linearly with $x$ (see Figs. 7a,b). The reason for this dependence must be found in the complex exponential in Eq. (32), which results in $\phi_{\perp}=-\beta x$. A linear fit to Fig. 7a gives $\beta=0.8806 / L$, whereas substituting $\theta=10^{\circ}$ and $k_{y} L=5$ in Eq. (33) yields $\beta=0.8816 / L$. Once more an excellent agreement between the numerical and analytical results is found. Furthermore, cuts of $\phi_{\perp}$ across magnetic surfaces (Fig. 7c) display a $\pi$ phase jump at the singular layer, which is reminiscent of the change of sign of $v_{\perp}$ caused by the $1 / s$ term when either $k_{y}$ or $B_{y}$ are zero (Figs. 5b,c). On the other hand, $\phi_{\mathrm{n}}$ does not suffer that jump and is quite smooth across the resonant surface (Fig. 7d).

\subsection{Fast modes}

Just like with Alfvén continuum modes, the presence of a longitudinal magnetic field component and/or a longitudinal wavenumber produces changes in the discrete, global fast modes of the system. Arregui et al. (2001) showed that in a straight, uniform magnetic field configuration with $B_{y}=0$ and $k_{y}=0$ fast mode solutions are characterised by $v_{\perp}=0$ and $v_{\mathrm{n}}$ displaying a smooth spatial structure determined by the numbers $n_{x}$ and $n_{z}$ of extrema in the $x$ - and $z$-directions. This number of extrema also determines the oscillatory frequency of fast modes.

Such as has been previously stated, to distinguish between the effect of mode coupling from the effects of $B_{y} \neq 0$ and $k_{y} \neq$ 0 , it is necessary to consider fast modes whose frequency lies outside the Alfvén continuum. For this reason, we plot together the frequencies of these two MHD modes as a function of the number of extrema along magnetic surfaces, $n_{x}$ (Fig. 8). Alfvén continuum modes have frequencies ranging from $\omega_{\min }$ to $\omega_{\max }$, which for $\delta>2$ are given by

$\frac{\omega_{\min } L}{v_{\mathrm{A} 0}}=k_{x} L=\frac{\pi}{2} n_{x}$

and

$\frac{\omega_{\max } L}{v_{\mathrm{A} 0}}=k_{x} L \exp \left[-\frac{(2-\delta)}{2} \frac{H}{L}\right]=\frac{\pi}{2} \exp \left[-\frac{(2-\delta)}{2} \frac{H}{L}\right] n_{x}$.

The above formulas have been calculated from the analytical expression (25) and taking into account the assumed equilibrium Alfvén speed, Eq. (4). Figure 8 shows that there are fast modes, e.g. the $n_{x}=n_{z}=1$ solution, whose frequency lies inside the Alfvén continuum for the same value of $n_{x}$, whereas other fast modes have frequencies that lie outside that continuum (for example the $n_{x}=1, n_{z}>2$ or the $n_{x}=2, n_{z}>4$ solutions). For this reason, two types of interaction between fast and Alfvén modes are expected, depending on whether the fast mode frequency is inside or outside the corresponding Alfvén continuum. We have found that the first kind of modes couple to Alfvén modes when either $B_{y} \neq 0$ or $k_{y} \neq 0$, while the second kind only couple to Alfvén modes when the equilibrium magnetic field has a longitudinal component. This mode coupling can be easily detected by means of the presence of a localised, non-zero perpendicular velocity component and a localised jump or singularity in the normal velocity component.

\subsubsection{Frequency}

We start by investigating the effect of $k_{y}$ on the frequency of fast modes outside the Alfvén continuum in a configuration with $\theta=0$ (see Fig. 9a). The increase of $\omega$ with $k_{y}$ 

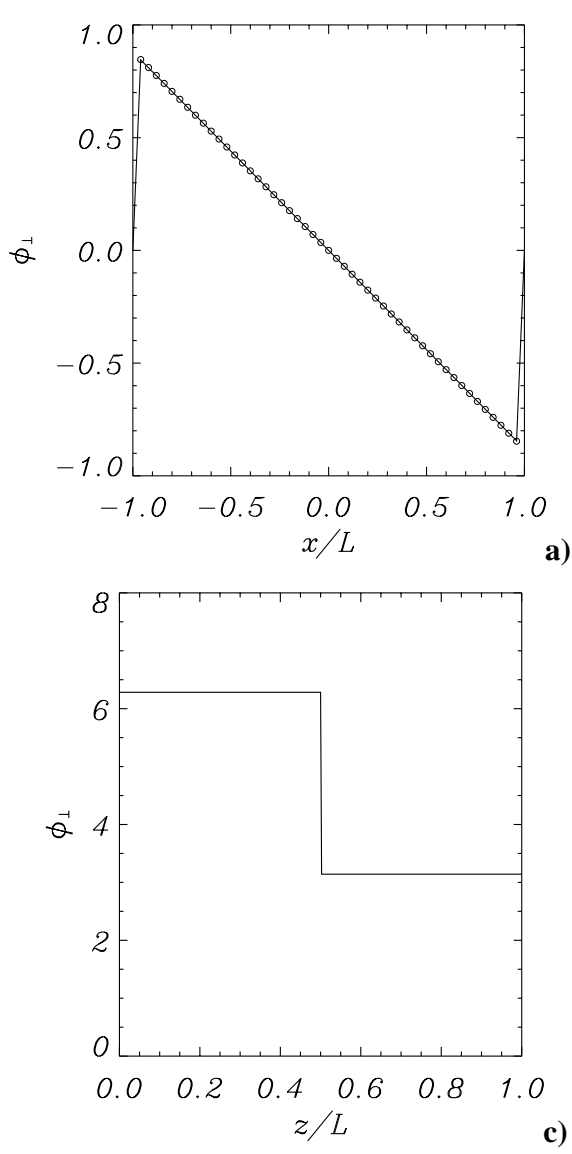
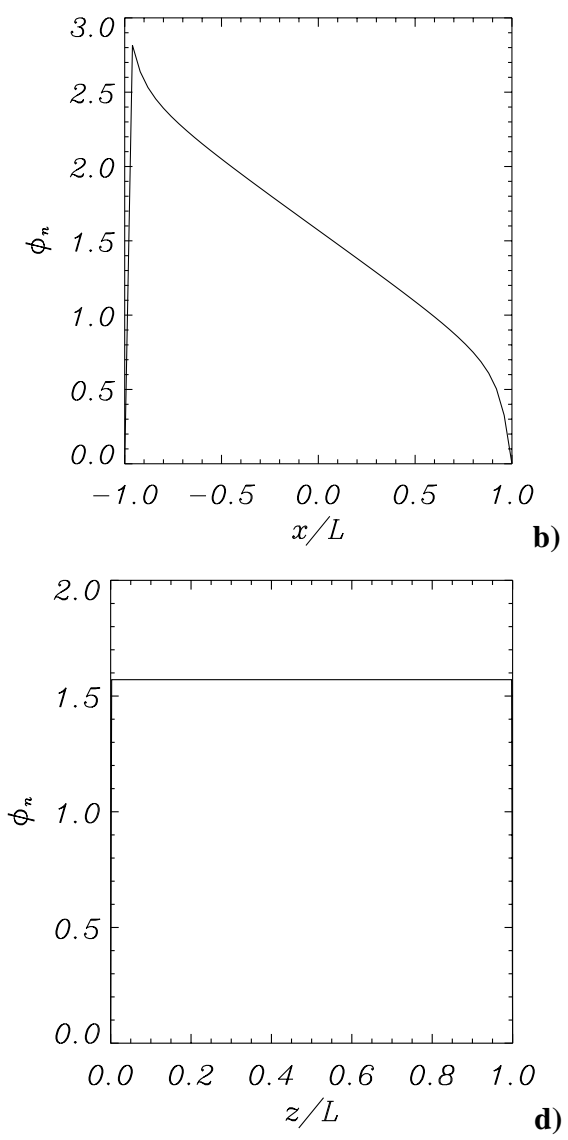

Fig. 7. a) and c) Cuts of $\phi_{\perp}$ and b) and d) cuts of $\phi_{\mathrm{n}}$ along $x=$ constant and $z=$ constant lines of an Alfvén mode for the parameter values $\theta=10^{\circ}, k_{y} L=5$ and $H / L=1$. In a) and b) the cuts are on the resonant surface, $z_{\mathrm{A}}=0.5 L$, while in c) and d) the cuts are along the $x=0$ direction. Circles in a) represent the phase $\phi_{\perp}=-\beta x$ derived from the analytical expression (32), with $\beta$ given by Eq. (33).

observed in this figure is the behaviour expected because of the property of fast modes being isotropic in nature. In a uniform and unbounded medium, this property results in the fast mode frequency being proportional to the sum of squares of the wavenumber components. To check whether this feature is preserved in the present bounded structure, the frequency squared has been plotted as a function of $k_{y}^{2}$ (Fig. 9b) and a fine confirmation of the linear relation between $\omega^{2}$ and $k_{y}^{2}$ is obtained.

The above results correspond to $B_{y}=0$ and so one would next consider an equilibrium with $B_{y} \neq 0$. Nevertheless, the magnetic field inclination, no matter how small, gives rise to a coupling between the fast mode and Alfvén continuum modes with similar frequency. Then, this kind of solutions are investigated in Sect. 4.3.

A final remark on the results plotted in Fig. 9 is in order. While the abovementioned relation between $\omega$ and $k_{y}$ is not surprising in a uniform, unbounded plasma (in which $\omega^{2}=v_{\mathrm{A}}^{2}\left(k_{x}^{2}+k_{y}^{2}+k_{z}^{2}\right)$ holds for fast modes), it is really an unexpected result in the present non-uniform, bounded configuration. Here, the $z$-dependence of the equilibrium variables is such that the fast MHD eigensolutions do not show a sinusoidal variation in the z-direction and, in addition, the Alfvén speed also changes with $z$, so an expression for $\omega$ like the previous one is meaningless. Despite all this, we see that the square of the frequency is still a linear function of $k_{y}^{2}$.

\subsubsection{Spatial structure}

Next, the change in the spatial structure of fast modes outside the Alfvén continuum caused by propagation along the longitudinal direction has been considered. When $k_{y}$ is gradually increased, $v_{\mathrm{n}}$ keeps its overall shape while $v_{\perp}$ acquires a larger amplitude, eventually exceeding that of the normal velocity component (Fig. 10). In addition, the spatial structure of these modes is smooth throughout the system and so they are uncoupled from localised Alfvén modes, since such a coupling shows up as jumps or singularities in $v_{\mathrm{n}}$ and $1 / s$ singularities in $v_{\perp}$ at certain magnetic surfaces. It must be noted that the value of $k_{y}$ at which the amplitudes of $v_{\mathrm{n}}$ and $v_{\perp}$ become equal is different for different fast modes.

The obtained decrease in the amplitude of $v_{\mathrm{n}}$ with respect to $v_{\perp}$ can have important implications from the observational point of view as fast modes could in principle be detected with the help of Doppler shifts by means of longitudinal motions in front view observations of coronal structures.

\subsection{Coupled fast modes and Alfvén modes}

\subsubsection{Frequency}

The frequencies of various fast modes that couple to Alfvén solutions (this coupling will be described in detail in Sect. 4.3.2) 


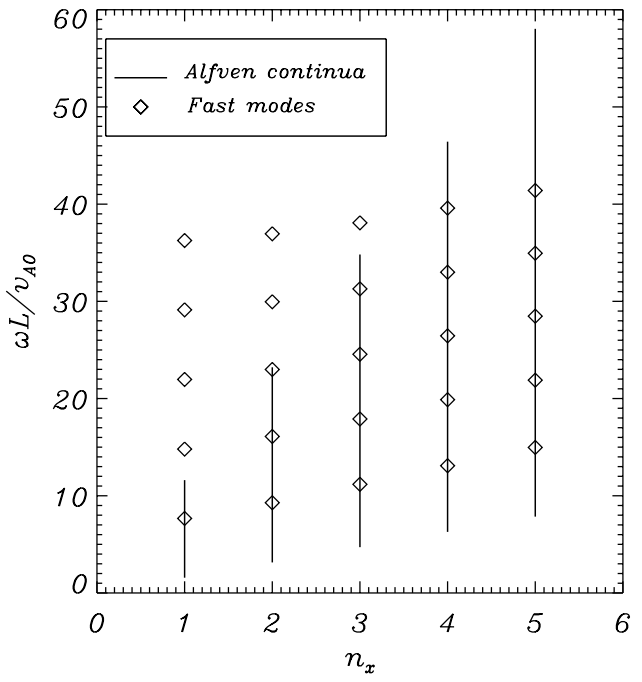

Fig. 8. Fast mode and Alfvén continuum frequencies in a purely poloidal magnetic field configuration $\left(B_{y}=0\right)$ with $\delta=6$ and $H / L=1$ and no longitudinal propagation $\left(k_{y}=0\right)$. For fast modes, the number of extrema of the eigenfunction $v_{\mathrm{n}}$ in the $x$-direction is $n_{x}$. For a fixed $n_{x}$ and with increasing frequency, the number of extrema of $v_{\mathrm{n}}$ in the vertical direction is $n_{z}=1,2, \ldots$ Only fast modes with $n_{z}$ between 1 and 5 are displayed.

are plotted in Fig. 11. From Figs. 11b, c it is obvious that the magnetic field orientation does not influence much the fast mode $\omega$. On the other hand, and contrary to the results in Sect. 4.2.1, the frequency of fast modes inside their own Alfvén continuum (like the $n_{x}=n_{z}=1$ or the $n_{x}=5, n_{z}=1$ ones) is not affected much by the longitudinal wavenumber (see Fig. 11a for $B_{y}=0$ and Fig. $11 \mathrm{~d}$ for $B_{y} \neq 0$ ).

Regarding the Alfvén modes in Fig. 11, it must be mentioned that only those that couple to the fast modes have been drawn. In addition, the fact of using a finite mesh to compute the solutions implies that only Alfvén modes whose resonant surface corresponds to one of the $z=$ constant lines of our computational grid are recovered. This means that, for example, the Alfvén modes in Fig. 11a are singular at the four different magnetic surfaces (from bottom to top) $z_{\mathrm{A}}=0.75 \mathrm{~L}, z_{\mathrm{A}}=0.766 \mathrm{~L}$, $z_{\mathrm{A}}=0.783 \mathrm{~L}$ and $z_{\mathrm{A}}=0.8 \mathrm{~L}$. Other solutions with singularities at intermediate values of $z$ can only be obtained by changing the grid spacing.

\subsubsection{Spatial structure}

We next investigate the influence on the spatial structure of fast modes of coupling with Alfvén continuum solutions. We have mentioned that such a coupling takes place (a) for all fast modes inside the Alfvén continuum with the same structure in the $x$-direction (i.e. with the same value of $n_{x}$ ) when either $k_{y} \neq 0$ or $B_{y} \neq 0$ and (b) for fast modes outside that continuum with $B_{y} \neq 0$.

We first choose the fast mode with $n_{x}=1$ and $n_{z}=1$ as representative of modes whose frequency falls into the limits of the Alfvén continuum in Fig. 8 and look at the effect of longitudinal wave propagation. Even for very small values of $k_{y}$, the structure of these modes is such that the perpendicular
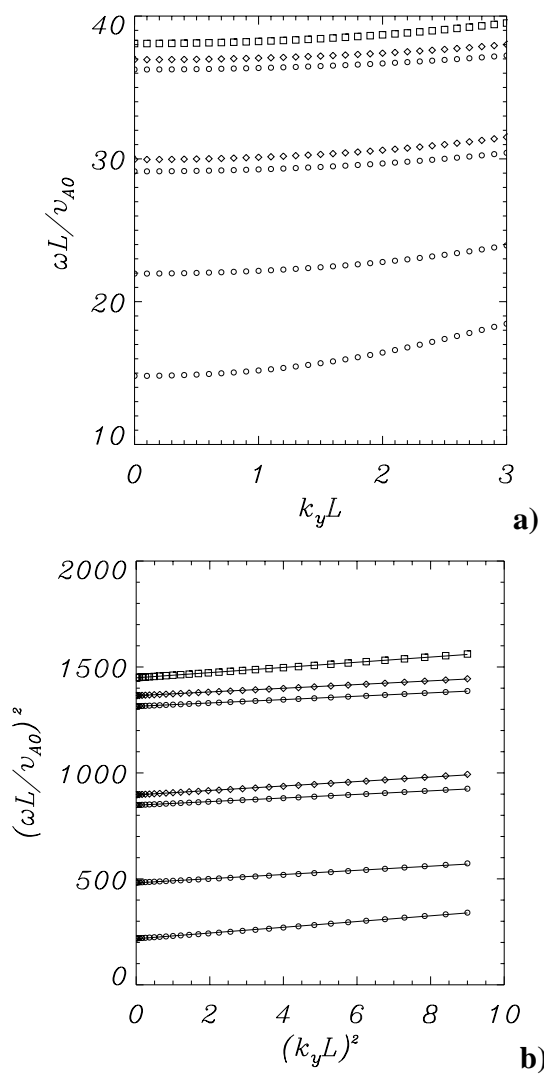

Fig. 9. a) Plot of the fast mode frequency as a function of the longitudinal wavenumber, $k_{y}$, for the four modes with $n_{x}=1$ and $n_{z}=2,3,4,5$ (circles), the two modes with $n_{x}=2$ and $n_{z}=4,5$ (diamonds) and the mode with $n_{x}=3$ and $n_{z}=5$ (squares). These modes lie outside the Alfvén continua with $n_{x}=1, n_{x}=2$ and $n_{x}=3$, see Fig. 8 . b) Same as in a) showing the linear relation that exists between $\omega^{2}$ and $k_{y}^{2}$. The continuous lines correspond to the best linear fit to the numerical values. Note that the frequency of fast modes depends more strongly on $n_{z}$ than on $n_{x}$. These solutions have been computed with a mesh of $N_{x}=N_{z}=61$ points.

velocity is much larger than the normal one and both functions display clear signs of the Alfvén mode to which the fast mode is coupled (see Figs. 12a, b). The Alfvén mode which couples to the fast mode is selected, amongst the $n_{x}=1$ continuum modes, as that whose frequency matches that of the global mode, which from Eq. (25) implies that a particular magnetic surface is selected and that the oscillatory velocity develops a singularity there. In particular, $v_{\mathrm{n}}$ contains a $\delta$-function contribution while $v_{\perp}$ displays a $1 / s$ contribution. In fact, the effect of mode coupling is so big that it is not possible to consider this solution a proper fast mode, since now the velocity is mostly confined to the vicinity of a magnetic surface and so the distribution is not even nearly isotropic. Moreover, there are two other modes (corresponding to the two Alfvén modes with highest $\omega$ in Fig. 11a) with similar frequency to the previous one and with almost the same spatial structure (one of them is presented in Figs. 12c, d). Owing to the $\delta$-function shape of $v_{\perp}$, the similarity between these solutions is here interpreted as the consequence of coupling between the Alfvén modes and the fast mode. Again, we are using the terms "fast" and "Alfvén" when dealing with these solutions because they originate from 

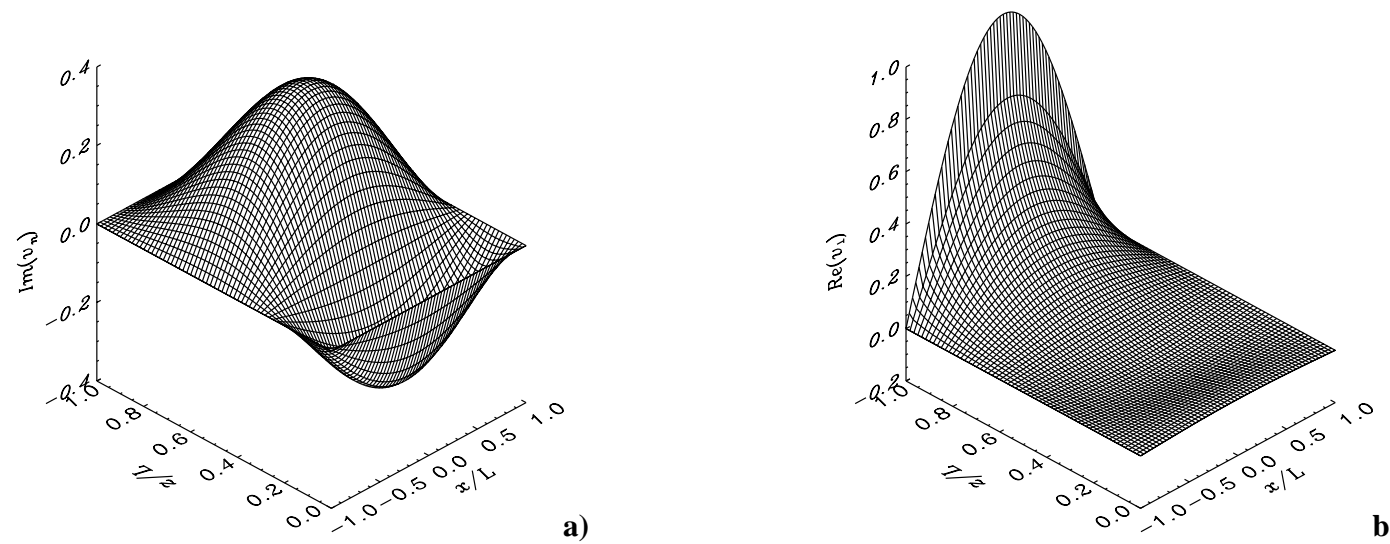

Fig. 10. a) Normal velocity component and b) perpendicular velocity component of the $n_{x}=1, n_{z}=2$ fast mode for $k_{y} L=1$ in an equilibrium with $\delta=6$ and $H / L=1$.
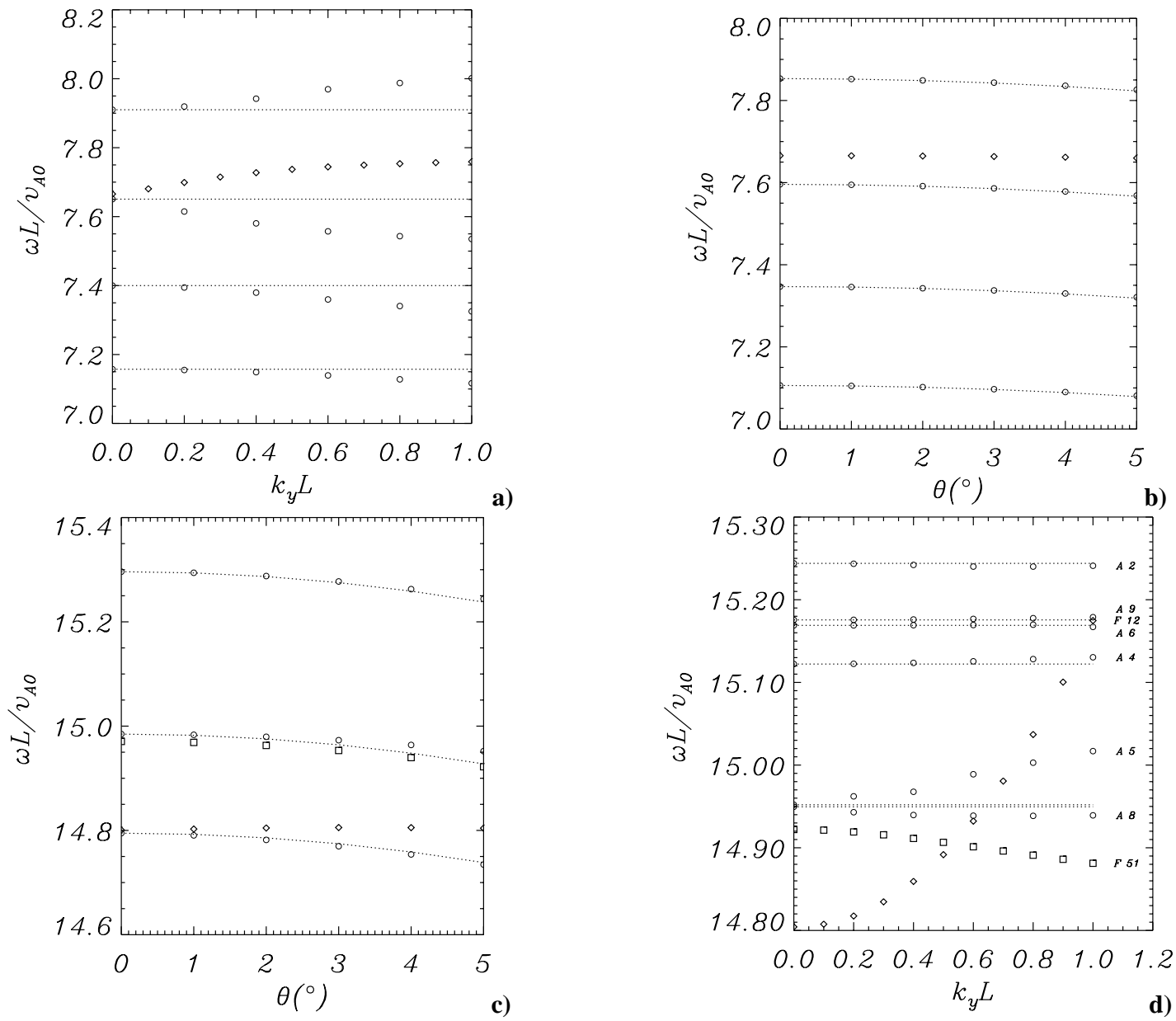

Fig. 11. Alfvén mode (circles) and fast mode (diamonds and squares) frequencies as a function of $k_{y}$ or the inclination angle, $\theta$. a) Fast mode with $n_{x}=n_{z}=1$ and the closest frequency Alfvén solutions for $B_{y}=0$. b) Same as a) for $k_{y}=0$. c) Fast modes with $n_{x}=1, n_{z}=2$ (diamonds) and with $n_{x}=5, n_{z}=1$ (squares) and the closest frequency Alfvén solutions $\left(k_{y}=0\right)$. d) Same as c) for $\theta=5^{\circ}$. The labels "F12" and "F51" indicate the position of the two fast modes in this diagram, whereas the labels "A $n_{x}$ " describe the position of Alfvén modes with $n_{x}$ extrema in the $x$-direction. In all these plots the dotted lines represent the analytical approximation to the Alfvén frequency, given by Eqs. (25) and (30). Large deviations of $\omega$ from the analytical value are in general an indication of strong coupling to a fast mode. In addition, only those Alfvén modes that couple to the fast modes are displayed.

such modes, despite they do not show their typical properties. In addition, to prove that none of these two modes is a proper Alfvén solution, the density perturbation is plotted for both of them in Fig. 13 and it is found that this variable takes finite values, contrary to what is expected for an Alfvén mode. 

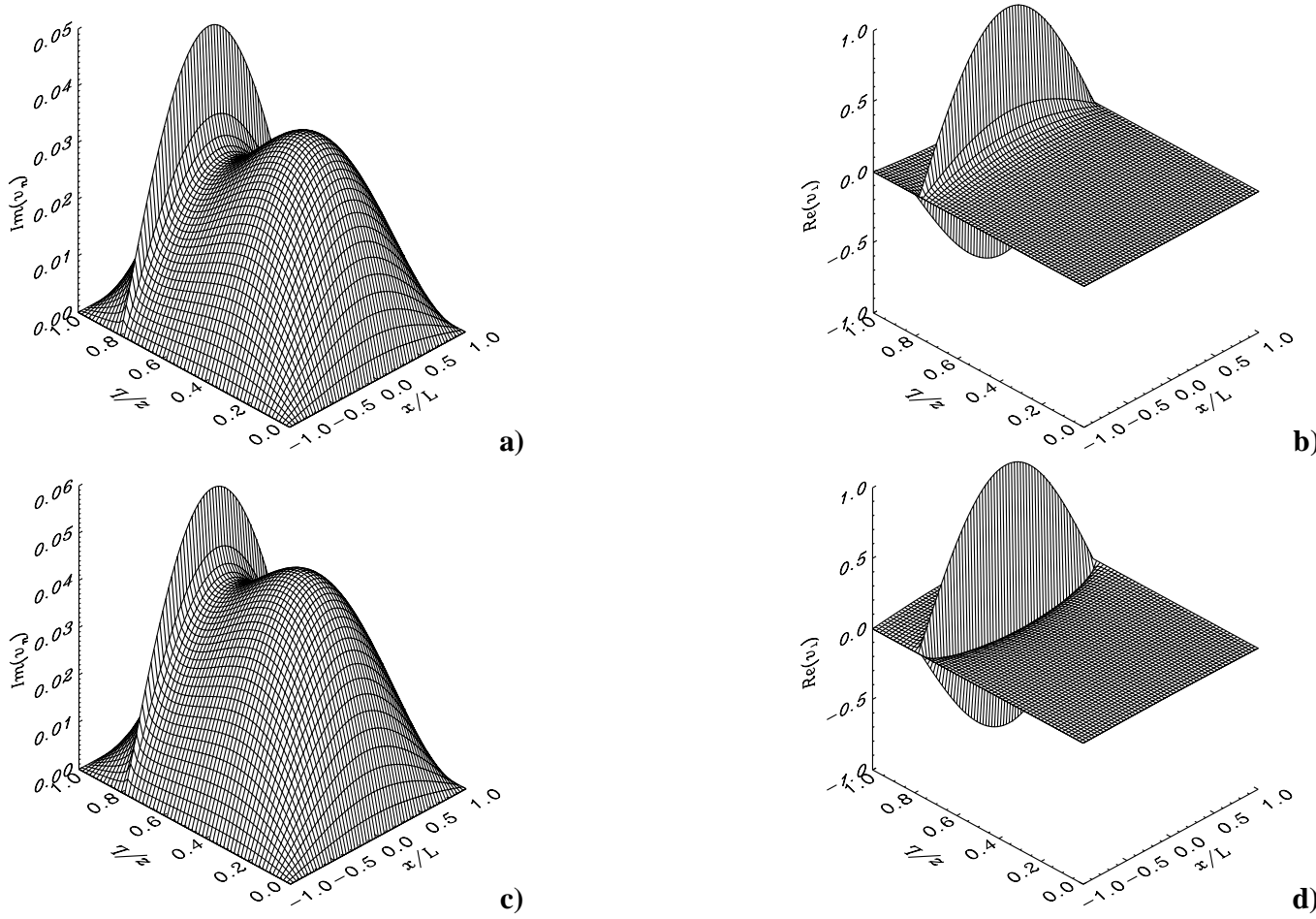

b)

d)

Fig. 12. This figure displays the solutions coming from the coupling between the $n_{x}=n_{z}=1$ fast mode and the Alfvén continuum modes with matching frequency and $n_{x}=1$ (the variation of the frequency of these modes with $k_{y}$ is shown in Fig. 11a). The reason for the coupling of the two waves is the non-zero longitudinal wavenumber $\left(k_{y} L=1\right)$ together with the fact that the original fast mode is inside the $n_{x}=1$ Alfvén continuum. a) Normal velocity component and b) perpendicular velocity component of the first mode. c) Normal and d) perpendicular velocity components of the second coupled mode. The equilibrium parameters are $\theta=0^{\circ}, \delta=6$ and $H / L=1$.
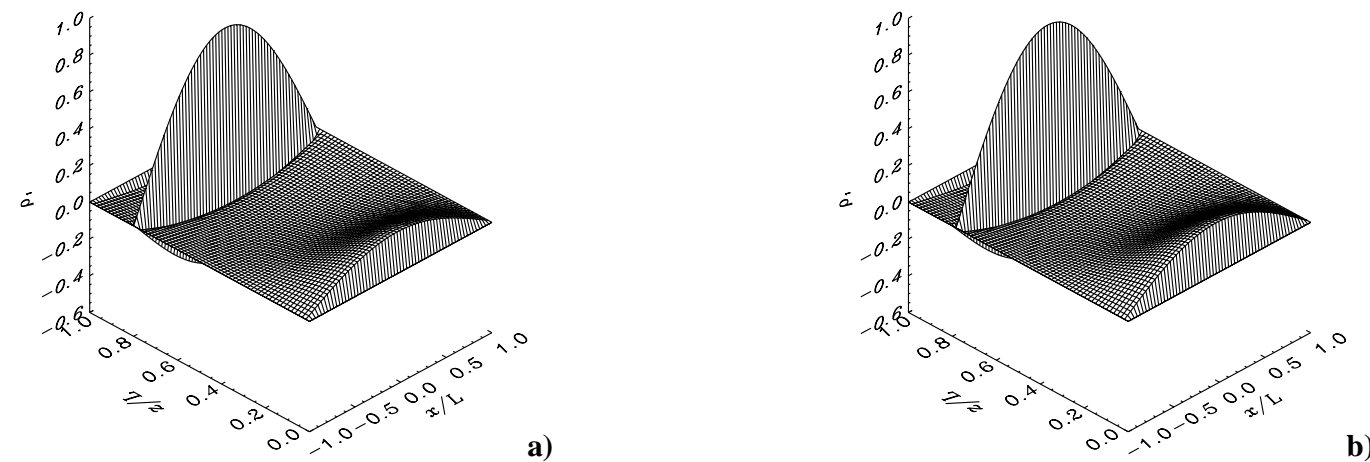

Fig. 13. Density perturbation for the modes displayed in a) Figs. 12a, b and b) Figs. 12c, d.

One may wonder why the above mode coupling only involves modes with the same parity about $x=0$. From Eqs. (11) and (12) with $B_{y}=0$ it is clear that opposite parities about $x=0$ in $v_{\mathrm{n}}$ and $v_{\perp}$ cannot couple together because those expressions contain second order derivatives with respect to $x$, but no first order derivatives with respect to this coordinate. As for the strength of the coupling, Fig. 11a shows that Alfvén mode frequencies are largely influenced by this effect, somehow behaving as if the "fast" mode "makes room" by pushing the "Alfvén" modes aside.

Now, the coupling of the $n_{x}=n_{z}=1$ solution to Alfvén modes caused by a non-zero longitudinal magnetic field component is addressed. Figure $11 \mathrm{~b}$ indicates that the coupling must be rather mild since Alfvén wave frequencies do not change much with $\theta$. This is well confirmed in Fig. 14, which shows $v_{\mathrm{n}}$ and $v_{\perp}$ for the "fast" and "Alfvén" modes (once more these terms only refer to the corresponding solutions for $k_{y}=0$ and $B_{y}=0$ ). The first mode (Figs. 14a, b) retains some fast mode features, like a roughly isotropic normal velocity component that is larger than the perpendicular one. Nevertheless, $v_{\perp}$ displays some oscillations confined to certain magnetic surfaces, characteristic of Alfvén waves. The second mode (Figs. 14c, d) is Alfvénic in essence, but its normal velocity component is smooth and global, although presenting a jump at the singular surface. The coupling now is between an "odd fast mode" (i.e. with odd parity of $v_{\mathrm{n}}$ about $x=0$ ) and a few "even Alfvén modes" (i.e. with even parity of $v_{\perp}$ about $x=0$ ), just as indicated by Eqs. (11) and (12) 

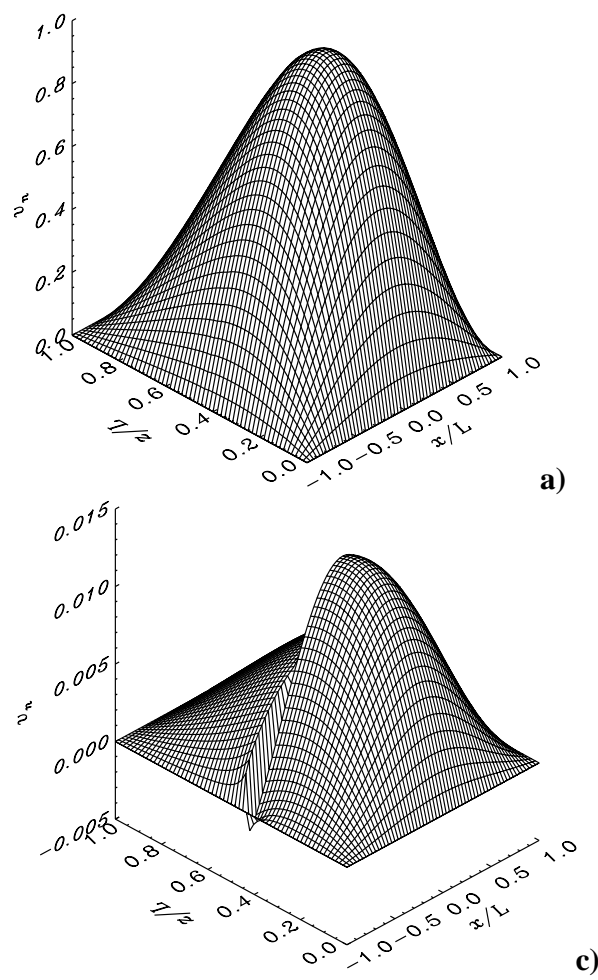
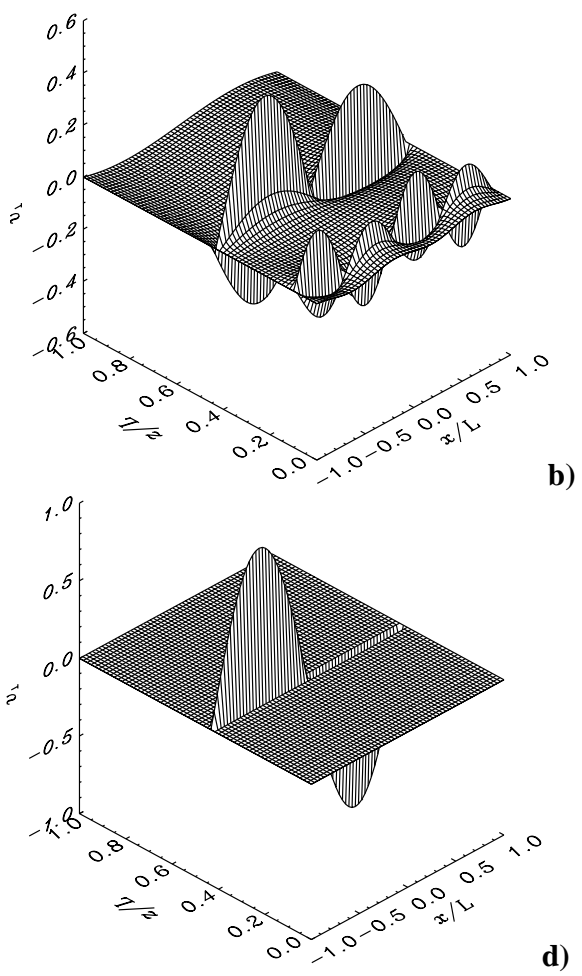

Fig. 14. Solutions coming from the coupling between the $n_{x}=1, n_{z}=1$ fast mode and the Alfvén continuum modes with matching frequency. a) Normal velocity component and b) perpendicular velocity component of the first mode. c) Normal and d) perpendicular velocity components of a second coupled mode. The main differences between the two modes are that the first one is associated to larger motions in the normal direction and that $v_{\perp}$ is smoother across the various singular layers. These solutions correspond to $k_{y} L=0$ with $\theta=5^{\circ}, \delta=6$ and $H / L=1$; the variation of their frequency with $\theta$ is shown in Fig. $11 \mathrm{~b}$.

with $k_{y}=0$, in which derivatives with respect to $x$ of $v_{\mathrm{n}}$ and $v_{\perp}$ are of opposite parity. The shape of $v_{\perp}$ in Fig. 14b evidences the influence of two Alfvén solutions with $n_{x}=2$ and $n_{x}=4$.

Next, we investigate mode coupling due to the longitudinal magnetic field for fast modes outside their own Alfvén continuum and choose the $n_{x}=1, n_{z}=2$ solution as a representative one. As soon as $B_{y} \neq 0$, the perpendicular velocity stops being zero and acquires values much larger than those of the normal component (Figs. 15a, b). Now, $v_{\mathrm{n}}$ is still a spatially smooth function, but the coupling to an Alfvén mode shows up through a small jump at a particular magnetic surface (around $z / L=0.8$ ). In addition, $v_{\perp}$ displays a structure characteristic of Alfvén modes, but instead of displaying one singular surface, there are a few of them easily visible. This is caused by the coupling of the fast mode with the $n_{x}=2,4,6,8$ Alfvén modes, which in order to match the fast mode frequency have different positions of the corresponding resonant surface. Moreover, the largest amplitude of $v_{\perp}$ is achieved at the singular surface where $v_{\mathrm{n}}$ has the above mentioned jump. Accompanying this mode one finds several solutions with very similar frequency and spatial dependence (one of them is plotted in Figs. 15c, d) which again comes from the Alfvén modes that couple to the fast mode. It must be emphasised that the use of the terms "fast" and "Alfvén" is not justified when referring to these modes since they both present a mixture of properties of both kind of waves.
The inclusion of longitudinal propagation does not change much the above picture (see Fig. 16). A few coupled modes exist with a frequency close to that of the original fast mode. The only differences worth mentioning are a smaller velocity amplitude in the perpendicular direction, a distortion in the shape of $v_{\mathrm{n}}$ and the signature in $v_{\perp}$ of Alfvén continuum modes with both even and odd number of extrema in the $x$-direction (oscillations with $n_{x}$ from 2 to 9 can be distinguished). Now, Eqs. (11) and (12) tell us that there are no restrictions about the parity of $v_{\mathrm{n}}$ and $v_{\perp}$ when both $k_{y}$ and $B_{y}$ are different from zero.

\section{Summary}

In this paper, the study of Arregui et al. (2001) on linear MHD waves for general force-free magnetic field configurations is continued. In that paper, a numerical code was presented for the study of linear MHD waves in general sheared, two-dimensional coronal magnetic structures including longitudinal propagation of the modes of oscillation. Rather than exciting waves by an initial perturbation or a continuous, periodic forcing, we concentrate on the normal modes of the system.

As a first step in this kind of study, a very simple magnetic configuration has been chosen consisting of a straight, inclined and uniform magnetic field. It has become evident that our original numerical code is not suitable for the study of fast and Alfvén waves whenever $B_{y} \neq 0$ or $k_{y} \neq 0$ because the eigensolutions display spurious oscillations. For this reason, the 

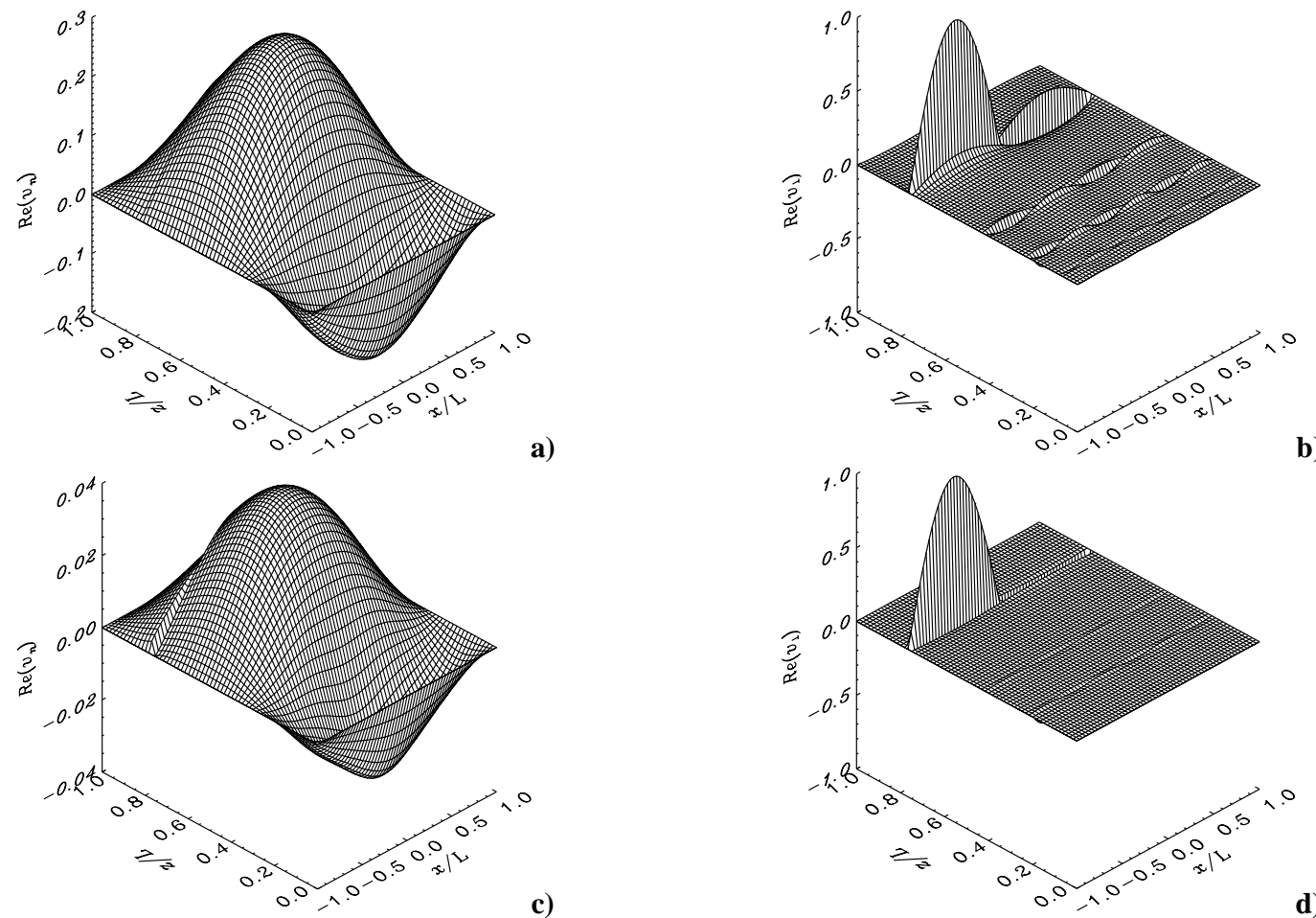

)

Fig. 15. Solutions coming from the coupling between the $n_{x}=1, n_{z}=2$ fast mode and the Alfvén continuum modes with matching frequency. a) Normal velocity component and b) perpendicular velocity component of the first mode. c) Normal and d) perpendicular velocity components of a second coupled mode. The main differences between the two modes are that the first one is associated to larger motions in the normal direction and that $v_{\perp}$ is smoother across the various singular layers. These solutions correspond to $k_{y} L=0, \theta=5^{\circ}, \delta=6$ and $H / L=1$; the variation of their frequency with $k_{y}$ and $\theta$ are shown in Figs. 11c, d, respectively.
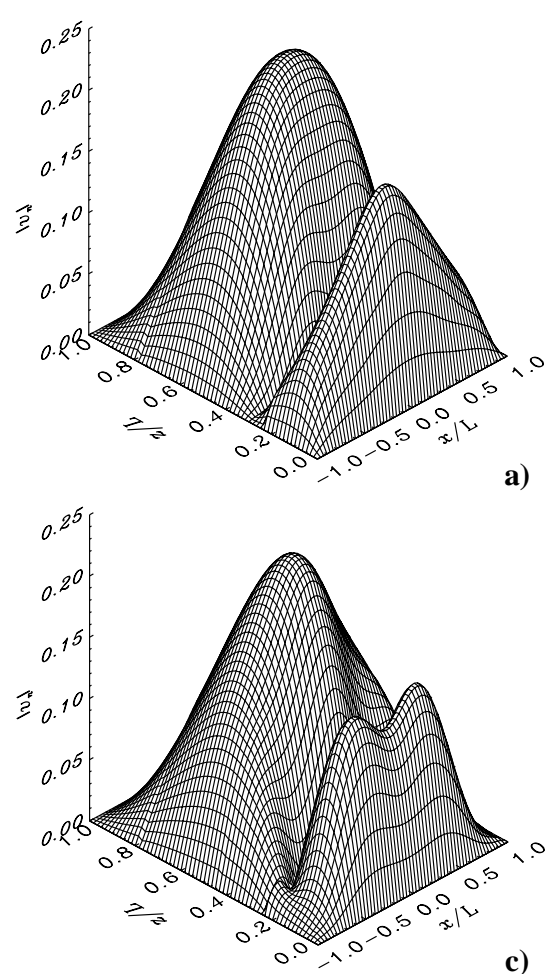
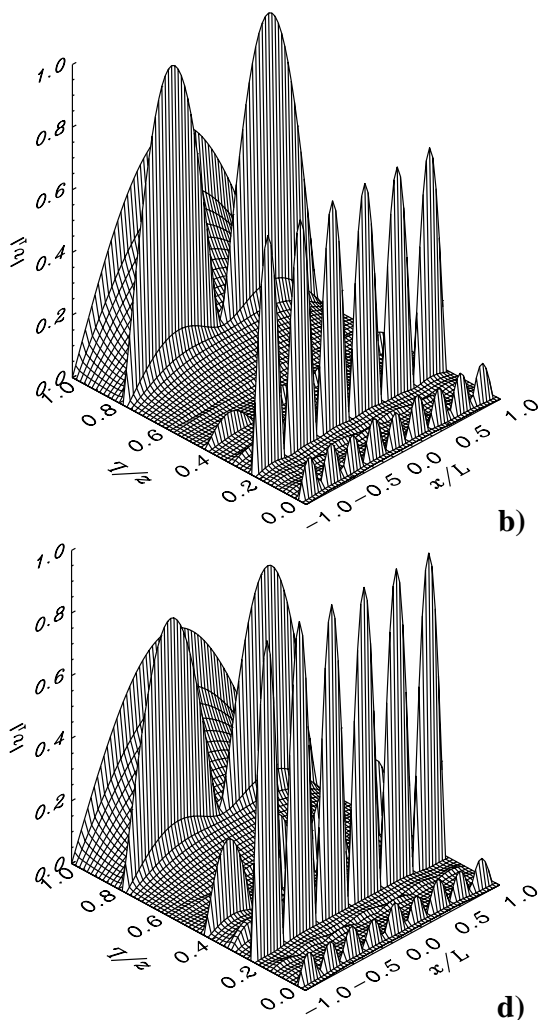

Fig. 16. Solutions coming from the coupling between the $n_{x}=1, n_{z}=2$ fast mode and the Alfvén continuum modes with matching frequency. a) Normal and b) perpendicular velocity components of the first mode. c) Normal and d) perpendicular velocity components of the second mode. There are only minor differences between the two modes, like the slight distortion of $v_{\mathrm{n}}$ for small $z$ in panel c). These solutions correspond to $k_{y} L=1$ with $\theta=5^{\circ}, \delta=6$ and $H / L=1$. 
numerical code has been modified in order to eliminate such oscillations by means of a staggered mesh arrangement.

A remarkable feature of the code is that the twodimensional spatial distribution of fast and Alfvén modes, together with the corresponding frequency, are obtained. In this way, and regarding Alfvén continuum modes, not only can the regular part of the solution be obtained, but also a good representation of the singular part. The code then proves to be a very good tool for obtaining solutions with either a global, smooth spatial structure or with singularities. Furthermore, an inspection of the frequencies of all the numerical solutions in this work reveals that their imaginary part is zero within machine precision. However, this does not mean that the imaginary part does not exist since asymptotic analysis around the resonant point in one-dimensional configurations has shown that the eigenvalue is complex and that the discrete modes with their frequencies in the Alfvén continuum are damped (Goedbloed 1983; Vanlommel et al. 2002).

Concerning the properties of Alfvén continuum modes, we see that longitudinal wave propagation $\left(k_{y} \neq 0\right)$ does not affect their eigenfrequencies. The inclusion of $B_{y} \neq 0$, however, has the effect of decreasing that oscillatory frequency by a factor $B_{x} / B=\cos \theta$, with $\theta$ the inclination angle, as a result of the increase of the length of field lines by the same factor $\cos \theta$. Apart from the oscillatory frequency, the spatial structure of Alfvén modes near the ideal singularity is also affected by the values of both $B_{y}$ and $k_{y}$. Non-zero values of these parameters change the singularity from a $\delta$ function-type to a $1 / s$-type. Concerning the regular part of the solution for Alfvén continuum modes, if only $B_{y}$ or $k_{y}$ are non-zero, the spatial structure remains unchanged with respect to the case in which both are zero. However, when both $k_{y} \neq 0$ and $B_{y} \neq 0$, the velocity components have non-zero real and imaginary parts and their regular part shows the ballooning contribution described in Halberstadt \& Goedbloed (1993). Nevertheless, if $v_{\perp}$ and $v_{\mathrm{n}}$ are considered in terms of their modulus and phase, it is seen that the regular part is again sinusoidal and that the phase changes linearly in the singular layer.

As for fast modes, it has been shown that only those whose frequency lies outside the corresponding Alfvén continuum spectrum for $B_{y}=0$ and $k_{y}=0$ are decoupled from Alfvén waves when longitudinal propagation is included. Quite surprisingly, the frequency of these fast modes varies linearly with $k_{y}^{2}$, just like in a homogeneous, unbounded plasma. Moreover, the spatial structure of these fast modes is quite global and so is not associated with particular field lines, but apart from the normal velocity component they also display a perpendicular component, which in some cases can be the dominant one. This means that this kind of solution has motions both in the normal and in the longitudinal direction.

All other fast modes (that is, those inside their own Alfvén continuum) couple to Alfvén modes with the same frequency when longitudinal propagation is included. Coupling between the two type of solutions is also present for an inclined magnetic field. This coupling leads to modes with mixed fast and Alfvén properties, such as $\delta$-function or $1 / s$ singularities in $v_{\perp}$ and Heaviside-like jumps or even $1 / s$ behaviour in $v_{\mathrm{n}}$ (similar to that in time-dependent simulations by Tirry et al. 1997;
De Groof et al. 2002; De Groof \& Goossens 2002). In addition, the perpendicular velocity component is often much larger than the normal one. Thus, when fast and Alfvén mode coupling takes place we end up with modes that, according to the spatial properties of the velocity, may resemble Alfvén continuum waves. Nevertheless, these modes show some exclusive properties of fast modes, such as non-zero density perturbations and a spatially global distribution of $v_{\mathrm{n}}$, and so must not be confused with Alfvén solutions.

From the observational point of view, the present results point out that the identification of modes present in simple magnetic configurations, such as the one in this work, is not an easy task. One may then wonder what problems are to be faced in the analysis of the oscillatory modes of real coronal objects, in which structuring and curvature play an important role.

Finally, we want to emphasise that the simple magnetic configuration studied in this paper constitutes a first step in the investigation of fast and Alfvén waves in more complex coronal structures, such as force-free, sheared coronal arcades.

Acknowledgements. The authors wish to acknowledge R. A. M. van der Linden, S. Poedts and W. J. Tirry for helpful comments and suggestions on the numerical scheme. I.A. is grateful to M. Goossens for his kind hospitality during his stay in Leuven. I.A. also acknowledges MCyT for a fellowship. The authors wish to acknowledge the financial support received from MCyT under project BFM2000-1329.

\section{References}

Ames, W. F. 1992, Numerical Methods for Partial Differential Equations, 3rd ed. (New York: Academic Press)

Arregui, I., Oliver, R., \& Ballester, J. L. 2001, A\&A, 369, 1122

Berghmans, D., \& Tirry, W. J. 1997, A\&A, 325, 318

De Groof, A., \& Goossens, M. 2002, A\&A, 386, 691

De Groof, A., Paes, K., \& Goossens, M. 2002, A\&A, 386, 681

Fletcher, C. A. J. 1988, Computational Techniques for Fluid Dynamics, Vol. II (Berlin-Heidelberg: Springer-Verlag)

Goedbloed, J. P. 1983, Lecture Notes on Ideal Magnetohydrodynamics (Rijhuizen Report), 83

Goedbloed, J. P., \& Halberstadt, G. 1994, A\&A, 286, 275

Goossens, M., \& Poedts, S. 1992, ApJ, 384, 348

Halberstadt, G., \& Goedbloed, J. P. 1993, A\&A, 280, 647

Hoffmann, K. A., \& Chiang, S. T. 1989, Computational Fluid Dynamics for Engineers, Vol. I (Austin, Texas: EES)

Mann, I. R., Wright, A. N., \& Cally, P. S. 1995, J. Geophys. Res., 100, 19441

Mitchell, A. R., \& Griffiths, D. F. 1980, The Finite Difference Method in Partial Differential Equations (New York: John Wiley \& Sons)

Oliver, R., Ballester, J. L., Hood, A. W., \& Priest, E. R. 1993, A\&A, 273,647

Oliver, R., Hood, A. W., \& Priest, E. R. 1996, ApJ, 461, 424

Ruderman, M. S., Goossens, M., Ballester, J. L., \& Oliver, R. 1997, A\&A, 328, 361

Tataronis, J. A., Torasso, R., \& Salat, A. 1998, Phys. Plasmas, 5, 3789

Terradas, J., Oliver, R., \& Ballester, J. L. 1999, ApJ, 517, 488

Tirry, W. J., \& Berghmans, D. 1997, A\&A, 325, 329

Tirry, W. J., Berghmans, D., \& Goossens, M. 1997, A\&A, 322, 329

Tirry, W. J., \& Poedts, S. 1998, A\&A, 329, 754

Vanlommel, P., Debosscher, A., Andries, J., \& Goossens, M. 2002, Sol. Phys., 205, 1

Wright, A. N., \& Rickard, G. J. 1995, ApJ, 444, 458 